\title{
LOW COMPLEXITY DAMPED GAUSS-NEWTON ALGORITHMS FOR CANDECOMP/PARAFAC
}

\author{
ANH HUY PHAN *, PETR TICHAVSKÝ ${ }^{\dagger}$, AND ANDRZEJ CICHOCKI $\stackrel{\ddagger}{\ddagger}$
}

\begin{abstract}
The damped Gauss-Newton (dGN) algorithm for CANDECOMP/PARAFAC (CP) decomposition can handle the challenges of collinearity of factors and different magnitudes of factors; nevertheless, for factorization of an $N$-D tensor of size $I_{1} \times \ldots \times I_{N}$ with rank $R$, the algorithm is computationally demanding due to construction of large approximate Hessian of size $(R T \times R T)$ and its inversion where $T=\sum_{n} I_{n}$. In this paper, we propose a fast implementation of the dGN algorithm which is based on novel expressions of the inverse approximate Hessian in block form. The new implementation has lower computational complexity, besides computation of the gradient (this part is common to both methods), requiring the inversion of a matrix of size $N R^{2} \times N R^{2}$, which is much smaller than the whole approximate Hessian, if $T \gg N R$. In addition, the implementation has lower memory requirements, because neither the Hessian nor its inverse never need to be stored in their entirety. A variant of the algorithm working with complex valued data is proposed as well. Complexity and performance of the proposed algorithm is compared with those of dGN and ALS with line search on examples of difficult benchmark tensors.
\end{abstract}

Key words. CP, tensor factorization, canonical decomposition, complex-valued tensor factorization, low-rank approximation, ALS, line search, Gauss-Newton, Levenberg-Marquardt, inverse problems

AMS subject classifications. 15A69, 15A23, 15A09, 15A29

1. Introduction. Algorithms for canonical polyadic decomposition, also coined CANDECOMP/PARAFAC (CP), can work well for general data [3, 14, 16]. However, they often fail for data with factors of different magnitudes [20] or collinear factors such as bottlenecks and swamps. Bottlenecks arise when two or more components are collinear [6, 9], and swamps arise when collinearity exists in all modes [6, 17]. Alternating least squares (ALS) algorithms with line searches, regularization, and rotation can improve performance, but they do not completely solve the problems. The damped Gauss-Newton (dGN) or LevenbergMarquardt (LM) algorithm has been confirmed to successfully decompose such difficult data [11, 19, 21, 29,31]. However, because these methods require the inverse of a large-scale approximate Hessian matrix, the dGN algorithm is not applicable to real-world large-scale and high-dimensional data. In this paper, we establish a fast inverse of the approximate Hessian for low-rank tensor factorization by proving that the approximate Hessian for low-rank tensor factorization is a low-rank adjustment to a block diagonal matrix, and propose fast dGN algorithms that do not need to store the approximate Hessian and its inverse entirely at one time.

The paper is organized as follows. Notation and basic multilinear algebra are briefly reviewed in Section 2 CP model and common algorithms are shortly reviewed in Section 3 Section 4 derives the fast dGN algorithm. Low-rank adjustment of approximate Hessian is derived, and its fast inverse is deduced in this section. The fast dGN algorithm with two variants has been proposed in Section 4.2. The fast dGN is extended to complex-valued tensor factorization in Section 5. In Section 6 we provide examples illustrating the validity and performance of the proposed algorithms. Finally, Section 7 concludes the paper.

2. Tensor notation and CANDECOMP/PARAFAC (CP) model. We shall denote a tensor by bold calligraphic letters, e.g., $\mathcal{A} \in \mathbb{R}^{I_{1} \times I_{2} \times \cdots \times I_{N}}$, matrices by bold capital letters, e.g., $\mathbf{A}=\left[\boldsymbol{a}_{1}, \boldsymbol{a}_{2}, \ldots, \boldsymbol{a}_{R}\right] \in \mathbb{R}^{I \times R}$, and vectors by bold italic letters, e.g., $\boldsymbol{a}_{j}$ or $\boldsymbol{I}=\left[I_{1}, I_{2}, \ldots, I_{N}\right]$.

\footnotetext{
*Brain Science Institute, RIKEN, Wakoshi, Japan (phan@brain.riken.jp).

${ }^{\dagger}$ Institute of Information Theory and Automation, Prague, Czech (tichavsk@utia.cas.cz). The work of P. Tichavský was supported by Ministry of Education, Youth and Sports of the Czech Republic through the project 1M0572 and by Grant Agency of the Czech Republic through the project 102/09/1278.

¥Brain Science Institute, RIKEN, Wakoshi, Japan (cia@brain.riken.jp).
} 
Mode- $n$ tensor unfolding of $\boldsymbol{y}$ is denoted by $\mathbf{Y}_{(n)}$. Generally, we adopt notation used in [5, 14]. The Kronecker, Khatri-Rao (column-wise Kronecker) and Hadamard products and are denoted respectively by $\otimes, \odot, \circledast$, [5, 14].

Notation 2.1. Given $N$ matrices $\mathbf{A}^{(n)} \in \mathbb{R}^{I_{n} \times R}$, we consider the following products

$$
\begin{aligned}
& \circledast_{n=1}^{N} \mathbf{A}^{(n)}=\mathbf{A}^{(N)} \circledast \cdots \circledast \mathbf{A}^{(n)} \circledast \cdots \circledast \mathbf{A}^{(1)}, \quad I_{n}=I, \forall n, \\
& \circledast \mathbb{A}_{k \neq n} \mathbf{A}^{(k)}=\mathbf{A}^{(N)} \circledast \cdots \circledast \mathbf{A}^{(n+1)} \circledast \mathbf{A}^{(n-1)} \circledast \cdots \circledast \mathbf{A}^{(1)}, \quad I_{n}=I, \forall n, \\
& \bigodot_{k \neq n} \mathbf{A}^{(k)}=\mathbf{A}^{(N)} \odot \cdots \odot \mathbf{A}^{(n+1)} \odot \mathbf{A}^{(n-1)} \cdots \odot \mathbf{A}^{(1)} .
\end{aligned}
$$

Definition 2.1. (Partitioned matrix and block matrix) A partitioned matrix $\mathbf{U}$ of $N$ matrices $\mathbf{U}^{(n)}$ along the mode-2 (horizontal) is denoted by

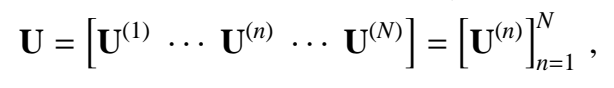

and a partitioned matrix $\mathbf{V}$ of NM matrices $\mathbf{V}^{(n, m)}$ along two modes is denoted by $\mathbf{V}=$ $\left[\mathbf{V}^{(n, m)}\right]_{n=1, m=1}^{N, M}$. A block diagonal matrix $\mathbf{B}$ of $N$ matrices $\mathbf{U}^{(n)}$ is denoted by

$$
\mathbf{B}=\left[\begin{array}{lll}
\mathbf{U}^{(1)} & & \\
& \ddots & \\
& & \mathbf{U}^{(N)}
\end{array}\right]=\operatorname{blkdiag}\left(\mathbf{U}^{(1)}, \cdots, \mathbf{U}^{(N)}\right)=\operatorname{blkdiag}\left(\mathbf{U}^{(n)}\right)_{n=1}^{N} .
$$

DefinItion 2.2. (CANDECOMP/PARAFAC (CP)) A CPD consists in representing a given $N$-th order data tensor $\boldsymbol{y} \in \mathbb{R}^{I_{1} \times I_{2} \times \cdots \times I_{N}}$ by a set of $N$ matrices (factors): $\mathbf{A}^{(n)}=$ $\left[\boldsymbol{a}_{1}^{(n)}, \boldsymbol{a}_{2}^{(n)}, \ldots, \boldsymbol{a}_{R}^{(n)}\right] \in \mathbb{R}^{I_{n} \times R},(n=1,2, \ldots, N)[4] 10$ ] 12 such that

$$
\boldsymbol{y} \approx \sum_{r=1}^{R} \boldsymbol{a}_{r}^{(1)} \circ \boldsymbol{a}_{r}^{(2)} \circ \ldots \circ \boldsymbol{a}_{r}^{(N)}=\widehat{\boldsymbol{y}},
$$

where symbol "o" denotes outer product. Tensor $\widehat{y}$ is an approximation of the data tensor $y$. We often assume unit-length components $\left\|\boldsymbol{a}_{r}^{(n)}\right\|_{2}=1$ for $n=1,2, \ldots, N-1, r=1,2, \ldots, R$.

3. CP Algorithms. The Alternating Least Squares (ALS) algorithm [2-4, 10,33] sequentially updates $\mathbf{A}^{(n)}$ using the update rule given by

$$
\mathbf{A}^{(n)}=\mathbf{Y}_{(n)}\left(\bigodot_{k \neq n} \mathbf{A}^{(k)}\right)\left(\boldsymbol{\Gamma}^{(n)}\right)^{\dagger}, \quad(n=1,2, \ldots, N),
$$

where $\boldsymbol{\Gamma}^{(n)}=\circledast_{k \neq n} \mathbf{C}^{(k)}, \mathbf{C}^{(n)}=\mathbf{A}^{(n) T} \mathbf{A}^{(n)}(n=1,2, \ldots, N)$ is defined as in Notation 2.1 “†” denotes the pseudo-inverse.

Denote by $\mathfrak{a} \in \mathbb{R}^{R T}, T=\sum_{n} I_{n}$, concatenation of vectorizations of $\mathbf{A}^{(n)}, n=1,2, \ldots, N$,

$$
\mathfrak{a}=\left[\operatorname{vec}\left(\mathbf{A}^{(1)}\right)^{T} \cdots \operatorname{vec}\left(\mathbf{A}^{(n)}\right)^{T} \cdots \operatorname{vec}\left(\mathbf{A}^{(N)}\right)^{T}\right]^{T}
$$

All-at-once algorithms such as the OPT algorithm [1], the PMF3, damped Gauss-Newton $(\mathrm{dGN})$ algorithms [11,20,29,31] simultaneously update $\mathfrak{a}$. The dGN algorithm is given by

$$
\begin{aligned}
\mathfrak{a} & \leftarrow \mathfrak{a}+\left(\mathbf{H}+\mu \mathbf{I}_{R T}\right)^{-1} \boldsymbol{g}, \\
\mathbf{H} & =\mathbf{J}^{T} \mathbf{J}, \quad \boldsymbol{g}=\mathbf{J}^{T} \operatorname{vec}(\mathcal{E}) .
\end{aligned}
$$

where $\mathcal{E}=\boldsymbol{y}-\widehat{\boldsymbol{y}}, \mathbf{J} \in \mathbb{R}^{J \times R T},\left(J=\prod_{n} I_{n}\right)$ is the Jacobian of vec $(\widehat{\boldsymbol{y}})$ with respect to $\mathfrak{a}, \mathbf{H}$ denotes the approximate Hessian, and the damping parameter $\mu>0$. Paatero [20] emphasized advantage of dGN compared with ALS when dealing with problems regarding swamps, different magnitudes of factors. 
The Gauss-Newton (GN) algorithm can be derived from Newton's method. Hence, the rate of convergence of the update rule (3.3) is at most quadratic. However, these methods face problems involving the large-scale Jacobian and large-scale inverse of the approximate Hessian $\mathbf{H}=\mathbf{J}^{T} \mathbf{J} \in \mathbb{R}^{R T \times R T}$. In order to eliminate the Jacobian, Paatero [20] established explicit expressions for submatrices of $\mathbf{H}$. We note that inverse of $\mathbf{H}$ is the largest workload of the GN algorithm with a complexity of order $O\left(R^{3} T^{3}\right)$ besides the computation of the gradient $\boldsymbol{g}$. Paatero [20] solved the inverse problem $\mathbf{H}^{-1}$ by Cholesky decomposition of the approximate Hessian and back substitution. However, the algorithm is still computationally demanding. Tomasi [29] extended Paatero's results [20], and derived a convenient method to construct $\mathbf{H}$ and the gradient for $N$-way tensor without using the Jacobian. In order to cope with the inverse of $\mathbf{H}$, Tomasi [30] used QR decomposition. However, the efficiency of existing dGN algorithms are still not sufficient for the large-scale problems due to the inverse $\mathbf{H}^{-1}$.

Recently, Tichavský and Koldovský [24] have proposed a novel method to invert the approximate Hessian based on $3 R^{2} \times 3 R^{2}$ dimensional matrices. For low-rank approximation $R \ll I_{n}, \forall n$, this method dramatically improves the running time. However, the algorithm still demands significant temporary extra-storage, and it is restricted for third-order tensors.

4. Fast damped Gauss-Newton algorithm. In this section, we will derive a fast dGN algorithm for low-rank approximation of tensors with arbitrary dimensions. The most important challenge of the update rule (3.3) is to reduce the computational cost for construction of the approximate Hessian $\mathbf{H}$ and its inverse.

Theorem 4.1 (Fast dGN algorithm). Define matrices $\boldsymbol{\Gamma}^{(n, m)}$ of size $(R \times R), n=1,2, \ldots, N$, $m=1,2, \ldots, N$, and a partitioned matrix $\mathbf{K}$ of size $\left(N R^{2} \times N R^{2}\right)$ comprising matrices $\mathbf{K}^{(n, m)}$

$$
\begin{aligned}
& \boldsymbol{\Gamma}^{(n, m)}=\left[\boldsymbol{\Gamma}^{(n, m)}\right]^{T}=\left[\boldsymbol{\Gamma}^{(m, n)}\right]^{T}=\underset{k \neq n, m}{\circledast} \mathbf{C}^{(k)}, \quad \mathbf{C}^{(n)}=\mathbf{A}^{(n) T} \mathbf{A}^{(n)} \quad \in \mathbb{R}^{R \times R}, \\
& \mathbf{K}^{(n, m)}=\left(1-\delta_{n, m}\right) \mathbf{P}_{R} \operatorname{diag}\left(\operatorname{vec}\left(\boldsymbol{\Gamma}^{(n, m)}\right)\right) \quad \in \mathbb{R}^{R^{2} \times R^{2}}, n=1, \ldots, N, m=1, \ldots, N,
\end{aligned}
$$

where $\delta_{n, m}$ is the Kronecker delta, $\mathbf{P}_{I, J}$ is a permutation matrix for any $I \times J$ matrix $\mathbf{X}$ such that $\mathbf{P}_{I, J} \operatorname{vec}\left(\mathbf{X}^{T}\right)=\operatorname{vec}(\mathbf{X}), \mathbf{P}_{R} \equiv \mathbf{P}_{R, R}$ and $\boldsymbol{\Gamma}^{(n)} \equiv \boldsymbol{\Gamma}^{(n, n)}$.

For $N R \ll T$, the fast dGN algorithm is written for each factor $\mathbf{A}^{(n)}$ as follows

$$
\mathbf{A}^{(n)} \leftarrow \mathbf{A}_{\mu}^{(n)}+\mathbf{A}^{(n)}\left(\mathbf{I}_{R}-\left(\mathbf{F}_{n}+\mathbf{\Gamma}^{(n)}\right) \widetilde{\boldsymbol{\Gamma}}_{\mu}^{(n)}\right), \quad n=1,2, \ldots, N,
$$

where $\mathbf{A}_{\mu}^{(n)}$ is a variant of the ALS update rule (3.1) with a damping parameter $\mu>0, \mathbf{F}_{n}$ of size $(R \times R)$ are frontal slices of $\mathcal{F}$ whose $\operatorname{vec}(\mathcal{F})=\mathbf{B}_{\mu} \boldsymbol{w}_{\mu}$, and

$$
\begin{aligned}
& \mathbf{A}_{\mu}^{(n)}=\mathbf{Y}_{(n)}\left(\bigodot_{k \neq n} \mathbf{A}^{(k)}\right) \widetilde{\boldsymbol{\Gamma}}_{\mu}^{(n)} \\
& \widetilde{\boldsymbol{\Gamma}}_{\mu}^{(n)}=\left(\boldsymbol{\Gamma}^{(n)}+\mu \mathbf{I}_{R}\right)^{-1}, \\
& \mathbf{B}_{\mu}=\left\{\begin{array}{ll}
\left(\mathbf{K}^{-1}+\boldsymbol{\Psi}_{\mu}\right)^{-1}, & \text { for invertible } \mathbf{K}, \\
\mathbf{K}\left(\mathbf{I}_{N R^{2}}+\Psi_{\mu} \mathbf{K}\right)^{-1}, & \text { otherwise, }
\end{array} \quad \mathbf{B}_{\mu} \in \mathbb{R}^{N R^{2} \times N R^{2}},\right. \\
& \boldsymbol{\Psi}_{\mu}=\operatorname{blkdiag}\left(\widetilde{\boldsymbol{\Gamma}}_{\mu}^{(n)} \otimes \mathbf{C}^{(n)}\right)_{n=1}^{N} \in \mathbb{R}^{N R^{2} \times N R^{2}}, \\
& \boldsymbol{w}_{\mu}=\operatorname{vec}\left(\left[\mathbf{A}^{(n) T} \mathbf{A}_{\mu}^{(n)}-\boldsymbol{\Gamma} \widetilde{\boldsymbol{\Gamma}}_{\mu}^{(n)}\right]_{n=1}^{N}\right) \quad \in \mathbb{R}^{N R^{2}}, \quad \boldsymbol{\Gamma}=\bigodot_{n=1}^{N} \mathbf{C}^{(n)} .
\end{aligned}
$$



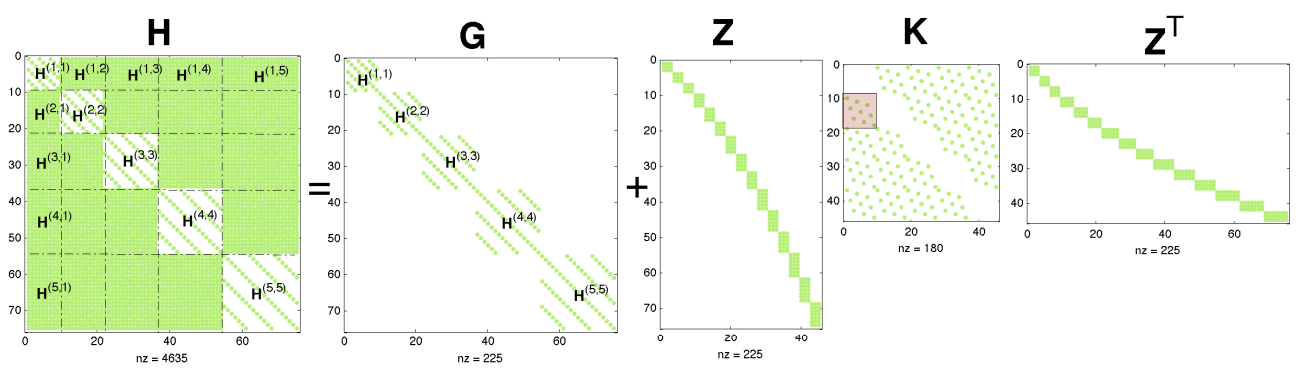

Fig. 4.1. Illustration of the approximate Hessian for a 5-D tensor which can be expressed as a low rank adjustment $\mathbf{H}=\mathbf{G}+\mathbf{Z} \mathbf{K} \mathbf{Z}^{T}$ as in Theorem 4.2] Green dots indicate nonzero elements.

In order to prove Theorem 4.1, we derive a low rank adjustment for $\mathbf{H}$ and employ the binomial inverse theorem [13] to invert a smaller matrix of size $N R^{2} \times N R^{2}$ instead of $\mathbf{H}^{-1}$.

\subsection{Fast inverse of the approximate Hessian $H$.}

Theorem 4.2 (Low rank adjustment for the approximate Hessian $\mathbf{H}$ ). With $\mathbf{K}$ defined in Theorem 4.1 the approximate Hessian $\mathbf{H}$ can be decomposed into

$$
\begin{array}{ll}
\mathbf{H}=\mathbf{G}+\mathbf{Z} \mathbf{K} \mathbf{Z}^{T}, & \\
\mathbf{G}=\operatorname{blkdiag}\left(\boldsymbol{\Gamma}^{(n)} \otimes \mathbf{I}_{I_{n}}\right)_{n=1}^{N} & \in \mathbb{R}^{R T \times R T}, \\
\mathbf{Z}=\operatorname{blkdiag}\left(\mathbf{I}_{R} \otimes \mathbf{A}^{(n)}\right)_{n=1}^{N} & \in \mathbb{R}^{R T \times N R^{2}} .
\end{array}
$$

Proof of Theorem 4.2 is given in Appendix B whereas an example of $\mathbf{H}$ for a 5-D tensor of size $3 \times 4 \times 5 \times 6 \times 7$ composed by 5 factors each of which has 3 components is illustrated in Fig. 4.1 In the left hand side of Fig. 4.1, $\mathbf{H}$ consists of $(N(N-1)) R^{2}$ rank-one matrices and $N R^{2}$ diagonal matrices which are located along its main diagonal.

TheOREM 4.3 (Fast inverse of the damped approximate Hessian). Inverse of the damped approximate Hessian $\mathbf{H}_{\mu}=\mathbf{H}+\mu \mathbf{I}_{R T}$ can be computed through

$$
\mathbf{H}_{\mu}^{-1}=\widetilde{\mathbf{G}}_{\mu}-\mathbf{L}_{\mu} \mathbf{B}_{\mu} \mathbf{L}_{\mu}^{T}
$$

where $\mathbf{B}_{\mu}$ is an $N R^{2} \times N R^{2}$ matrix defined in (4.6) and

$$
\begin{array}{ll}
\widetilde{\mathbf{G}}_{\mu}=\operatorname{blkdiag}\left(\widetilde{\boldsymbol{\Gamma}}_{\mu}^{(n)} \otimes \mathbf{I}_{I_{n}}\right)_{n=1}^{N} \quad \in \mathbb{R}^{R T \times R T}, \\
\mathbf{L}_{\mu}=\operatorname{blkdiag}\left(\widetilde{\boldsymbol{\Gamma}}_{\mu}^{(n)} \otimes \mathbf{A}^{(n)}\right)_{n=1}^{N} & \in \mathbb{R}^{R T \times N R^{2}} .
\end{array}
$$

The matrix $\mathbf{K}$ can also be expressed as a partitioned matrix of matrices $\mathbf{D}^{(n, m)}=(1-$ $\left.\delta_{n, m}\right) \operatorname{diag}\left(\operatorname{vec}\left(\boldsymbol{\Gamma}^{(n, m)}\right)\right) \in \mathbb{R}^{R^{2} \times R^{2}}$

$$
\mathbf{K}=\left(\mathbf{I}_{N} \otimes \mathbf{P}_{R}\right)\left[\mathbf{D}^{(n, m)}\right]_{n, m} .
$$

If all the entries $\gamma_{r, s}^{(n, m)}$ of $\boldsymbol{\Gamma}^{(n, m)}$ are non-zeros, the matrix $\mathbf{D}$ is invertible, and its inverse is also a partitioned matrix comprising diagonal matrices. Inverse of $\mathbf{K}$ is briefly described in Appendix E

An alternative expression $\mathbf{H}_{\mu}^{-1}$ can be written in block form.

THEOREM 4.4 (Fast inversion of $\mathbf{H}_{\mu}$ in the block form). Inverse of $\mathbf{H}_{\mu}$ can be written as 
where

$$
\mathbf{H}_{\mu}^{-1}=\widetilde{\mathbf{H}}_{\mu}=\left[\begin{array}{ccccc}
\widetilde{\mathbf{H}}_{\mu}^{(1,1)} & \ldots & \widetilde{\mathbf{H}}_{\mu}^{(1, m)} & \ldots & \widetilde{\mathbf{H}}_{\mu}^{(1, N)} \\
\vdots & \ddots & \vdots & \ddots & \vdots \\
\widetilde{\mathbf{H}}_{\mu}^{(n, 1)} & \ldots & \widetilde{\mathbf{H}}_{\mu}^{(n, m)} & \ldots & \widetilde{\mathbf{H}}_{\mu}^{(n, N)} \\
\vdots & \ddots & \vdots & \ddots & \vdots \\
\widetilde{\mathbf{H}}_{\mu}^{(N, 1)} & \ldots & \widetilde{\mathbf{H}}_{\mu}^{(N, m)} & \ldots & \widetilde{\mathbf{H}}_{\mu}^{(N, N)}
\end{array}\right],
$$

$$
\widetilde{\mathbf{H}}_{\mu}^{(n, m)}=\delta_{n, m}\left(\widetilde{\boldsymbol{\Gamma}}_{\mu}^{(n)} \otimes \mathbf{I}_{I_{n}}\right)-\left(\mathbf{I}_{R} \otimes \mathbf{A}^{(n)}\right) \widetilde{\mathbf{S}}_{\mu}^{(n, m)}\left(\mathbf{I}_{R} \otimes \mathbf{A}^{(m) T}\right),
$$

and $\widetilde{\mathbf{S}}_{\mu}^{(n, m)}=\left(\widetilde{\boldsymbol{\Gamma}}_{\mu}^{(n)} \otimes \mathbf{I}_{R}\right) \mathbf{B}_{\mu}^{(n, m)}\left(\widetilde{\boldsymbol{\Gamma}}_{\mu}^{(m)} \otimes \mathbf{I}_{R}\right)$ are matrices of size $R^{2} \times R^{2}$.

Proof. From (4.12), denote by $\mathbf{B}_{\mu}^{(n, m)}$ the $(m, n)$-th block of $\mathbf{B}_{\mu}$, we have

$$
\begin{aligned}
\widetilde{\mathbf{H}}_{\mu}^{(n, m)} & =\delta_{n, m}\left(\widetilde{\boldsymbol{\Gamma}}_{\mu}^{(n)} \otimes \mathbf{I}_{I_{n}}\right)-\left(\widetilde{\boldsymbol{\Gamma}}_{\mu}^{(n)} \otimes \mathbf{I}_{I_{n}}\right)\left(\mathbf{I}_{R} \otimes \mathbf{A}^{(n)}\right) \mathbf{B}_{\mu}^{(n, m)}\left(\mathbf{I}_{R} \otimes \mathbf{A}^{(m) T}\right)\left(\widetilde{\boldsymbol{\Gamma}}_{\mu}^{(m)} \otimes \mathbf{I}_{I_{n}}\right) \\
& =\delta_{n, m}\left(\widetilde{\boldsymbol{\Gamma}}_{\mu}^{(n)} \otimes \mathbf{I}_{I_{n}}\right)-\left(\mathbf{I}_{R} \otimes \mathbf{A}^{(n)}\right)\left(\widetilde{\boldsymbol{\Gamma}}_{\mu}^{(n)} \otimes \mathbf{I}_{R}\right) \mathbf{B}_{\mu}^{(n, m)}\left(\widetilde{\boldsymbol{\Gamma}}_{\mu}^{(m)} \otimes \mathbf{I}_{R}\right)\left(\mathbf{I}_{R} \otimes \mathbf{A}^{(m) T}\right) .
\end{aligned}
$$

Please note that the inversion of $\mathbf{H}_{\mu}$ in the block form saves memory. It requires to save only the matrices $\widetilde{\boldsymbol{\Gamma}}_{\mu}^{(n)}$ and $\widetilde{\mathbf{S}}_{\mu}$. While the full matrix $\mathbf{H}$ or its inverse has $R^{2} T^{2}$ elements, the memory saving format only requires to store $N R^{2}$ elements of matrices $\widetilde{\boldsymbol{\Gamma}}_{\mu}^{(n)}$ and $N^{2} R^{4}$ elements of $\widetilde{\mathbf{S}}_{\mu}$. प

4.2. Proof of Theorem 4.1. We replace $\mathbf{H}_{\mu}^{-1}$ in (3.3) by those in (4.12) in Theorem 4.3 or Theorem 4.4 and formulate the fast dGN algorithm

$$
\mathfrak{a} \leftarrow \mathfrak{a}+\widetilde{\mathbf{G}}_{\mu} \mathbf{g}-\mathbf{L}_{\mu} \mathbf{B}_{\mu} \mathbf{L}_{\mu}^{T} \mathbf{g} .
$$

The Jacobian, which may demand high computational cost, still exists in the gradient $\boldsymbol{g}$ in the update rule (4.18). We also note that $\mathbf{L}_{\mu}$ is a block diagonal matrix of $N$ Kronecker products $\left(\widetilde{\boldsymbol{\Gamma}}_{\mu}^{(n)} \otimes \mathbf{A}^{(n)}\right) \in \mathbb{R}^{R I_{n} \times R^{2}}$ given in (4.14). Construction of $\mathbf{L}_{\mu}$ has a computational complexity of order $O\left(T R^{3}\right)$, and requires an extra-storage of $O\left(T R^{3}\right)$. In order to completely bypass the Jacobian $\mathbf{J}$ in (4.18) and avoid building up the matrix $\mathbf{L}_{\mu}$, we seek convenient methods for computing $\widetilde{\mathbf{G}}_{\mu} \boldsymbol{g}, \boldsymbol{w}_{\mu}=\mathbf{L}_{\mu}^{T} \boldsymbol{g}$, and product $\mathbf{L}_{\mu} \mathbf{B}_{\mu} \boldsymbol{w}_{\mu}$.

Lemma 4.5 (Optimize the update rule (4.18)). With $\mathbf{A}_{\mu}^{(n)}, \boldsymbol{\Gamma}$ and the tensor $\mathcal{F}$ defined in Theorem 4.1]

$$
\begin{gathered}
\left(\widetilde{\mathbf{G}}_{\mu} \boldsymbol{g}\right)^{T}=\left[\operatorname{vec}\left(\mathbf{A}_{\mu}^{(n)}-\mathbf{A}^{(n)} \mathbf{\Gamma}^{(n)} \widetilde{\boldsymbol{\Gamma}}_{\mu}^{(n)}\right)^{T}\right]_{n=1}^{N}, \\
\boldsymbol{w}_{\mu}=\mathbf{L}_{\mu}^{T} \boldsymbol{g}=\operatorname{vec}\left(\left[\mathbf{A}^{(n) T} \mathbf{A}_{\mu}^{(n)}-\mathbf{\Gamma} \widetilde{\boldsymbol{\Gamma}}_{\mu}^{(n)}\right]_{n=1}^{N}\right), \\
\mathbf{L}_{\mu} \mathbf{B}_{\mu} \boldsymbol{w}_{\mu}=\left[\begin{array}{c}
\operatorname{vec}\left(\mathbf{A}^{(1)} \mathbf{F}_{1} \widetilde{\boldsymbol{\Gamma}}_{\mu}^{(1)}\right) \\
\vdots \\
\operatorname{vec}\left(\mathbf{A}^{(n)} \mathbf{F}_{n} \widetilde{\boldsymbol{\Gamma}}_{\mu}^{(n)}\right) \\
\vdots \\
\operatorname{vec}\left(\mathbf{A}^{(N)} \mathbf{F}_{N} \widetilde{\boldsymbol{\Gamma}}_{\mu}^{(N)}\right)
\end{array}\right] .
\end{gathered}
$$




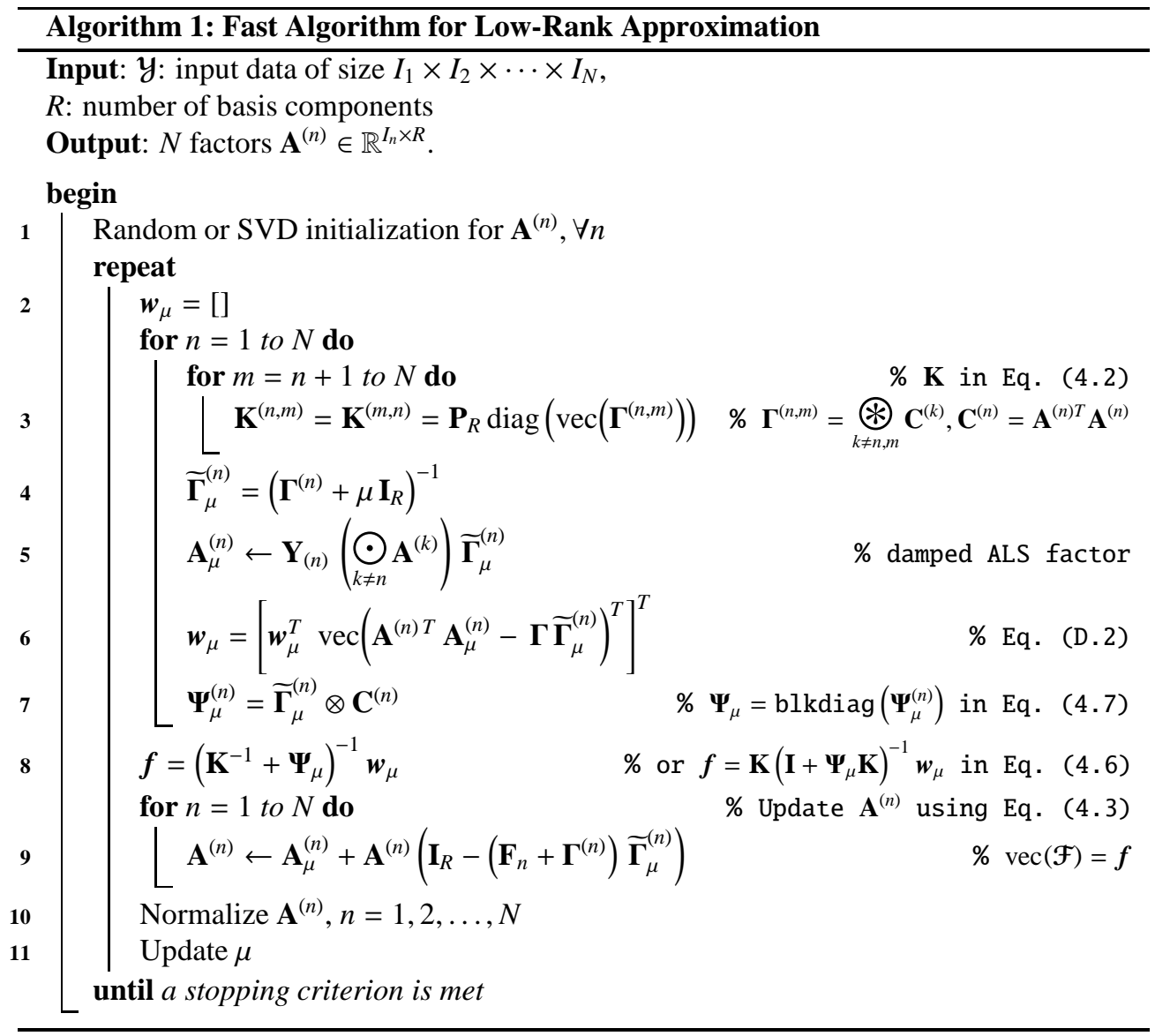

Proof of Lemma 4.5 is given in Appendix D By replacing $\widetilde{\mathbf{G}}_{\mu} \boldsymbol{g}, \mathbf{L}_{\mu}^{T} \boldsymbol{g}$, and $\mathbf{L}_{\mu} \mathbf{B}_{\mu} \boldsymbol{w}_{\mu}$ in (4.18) by those in (4.19), (4.20) and (4.21), we obtain a compact update rule for each factor $\mathbf{A}^{(n)}, n=1,2, \ldots, N$ as given in Theorem 4.1

We note that linear systems $\mathbf{B}_{\mu} \boldsymbol{w}_{\mu}$ in (4.6) have a computational complexity of order $O\left(N^{3} R^{6}\right)$ which is much lower than $O\left(R^{3} T^{3}\right)$ for $(\mathbf{H}+\mu \mathbf{I})^{-1}$ for $N R \ll T$. Pseudo code of the proposed algorithm based on the update rule (4.3) is given in Algorithm 1] If components of $\mathbf{A}^{(n)}$ are mutually non-orthogonal, $\mathbf{K}$ is invertible, and its inverse can be explicitly computed as in Appendix E In this case, Step 3 is replaced by (E.1). A practical normalization in Step 10 is that the energy of the components is equally distributed in all modes. The method often enhances the convergence speed of the LM iteration [32,33].

4.3. Two variants of the fast dGN algorithm. From (4.6), we present two variants of the fast dGN algorithm which solve the corresponding inverse problem $\boldsymbol{\Phi}^{-1} \boldsymbol{w}_{\mu}$.

(a) $\mathbf{f L M}_{\mathbf{a}}$. $\boldsymbol{\Phi} \triangleq \boldsymbol{\Phi}_{1}=\mathbf{I}_{N R^{2}}+\boldsymbol{\Psi}_{\mu} \mathbf{K}$ comprises $N$ diagonal matrices $\mathbf{I}_{R^{2}}$, and $N(N-1)$ block matrices $\left(\boldsymbol{\Gamma}^{(n)^{-1}} \otimes \mathbf{C}^{(n)}\right) \mathbf{P}_{R} \mathbf{D}^{(n, m)}$, for $n \neq m$. Note that $\boldsymbol{\Phi}_{1}$ is not symmetric, and its density is given by

$$
d_{\Phi_{1}}=\frac{N(N-1) R^{4}+N R^{2}}{N^{2} R^{4}}=\frac{(N-1) R^{2}+1}{N R^{2}} .
$$




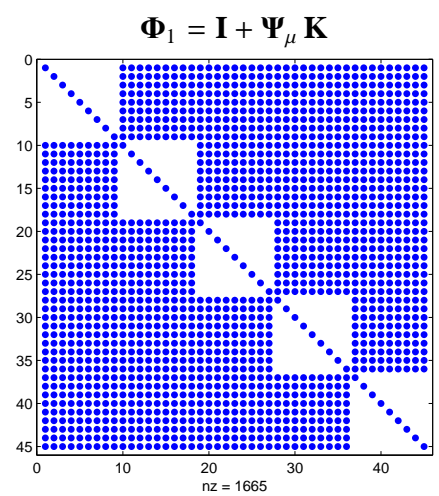

(a) $d_{\Phi_{1}}=\frac{(N-1) R^{2}+1}{N R^{2}}$.

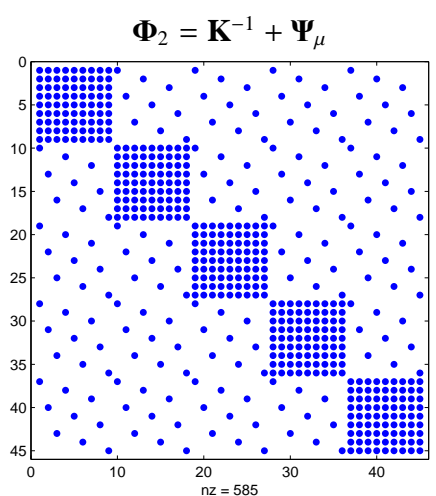

(b) $d_{\Phi_{2}}=\frac{R^{2}+N-1}{N R^{2}}$.

Fig. 4.2. Illustration of structure of $N R^{2} \times N R^{2}$ sparse matrices $\boldsymbol{\Phi}_{1}$ and $\boldsymbol{\Phi}_{2}$ for a $3 \times 4 \times 5 \times 6 \times 7$ dimensional tensor composed by $R=3$ rank-one tensors. The matrix $\boldsymbol{\Phi}_{1}$ is less sparse than the matrix $\boldsymbol{\Phi}_{2}$. Blue dots denote nonzero entries.

For 3-D tensor factorizations, the fast dGN algorithm in which Step 8 solves $\boldsymbol{\Phi}_{1}^{-1} \boldsymbol{w}_{\mu}$ simplifies into the LM-1 algorithm in [24].

(b) $\mathbf{f L M}_{\mathbf{b}} . \boldsymbol{\Phi} \triangleq \boldsymbol{\Phi}_{2}=\mathbf{K}^{-1}+\boldsymbol{\Psi}_{\mu}$ is a symmetric matrix of size $N R^{2} \times N R^{2}$ derived from (4.2) and (4.7). Theorem E.1 presents an explicit form of $\mathbf{K}^{-1}$ which is a partitioned matrix of $\left(R^{2} \times R^{2}\right)$ diagonal matrices. Hence, it has only $N^{2} R^{2}$ non-zero entries. The block diagonal matrix $\Psi_{\mu}$ (4.7) is constructed from $N\left(R^{2} \times R^{2}\right)$ sub-matrices. As a consequence, the density of the sparse matrix $\boldsymbol{\Phi}_{2} \in \mathbb{R}^{N R^{2} \times N R^{2}}$ is

$$
d_{\Phi_{2}}=\frac{N^{2} R^{2}+N R^{4}-N R^{2}}{N^{2} R^{4}}=\frac{R^{2}+N-1}{N R^{2}} .
$$

Because $\boldsymbol{\Phi}_{1}$ is not symmetric and less sparse than $\boldsymbol{\Phi}_{2}$, solving the linear system $\boldsymbol{\Phi}_{1}^{-1} \boldsymbol{w}_{\mu}$ could be more time consuming than solving $\boldsymbol{\Phi}_{2}^{-1} \boldsymbol{w}_{\mu}$. Inverse of $\mathbf{K}$ is not expensive and has the explicit expression given in Theorem E.1. However, when the factor matrices have mutually orthogonal columns, $\mathbf{K}$ is singular because it has collinear columns and rows. In Fig. 4.2, we illustrate the structures and properties of the two matrices $\boldsymbol{\Phi}_{1}$ and $\boldsymbol{\Phi}_{2}$ for a $3 \times 4 \times 5 \times 6 \times 7$ dimensional tensor composed by $R=3$ rank-one tensors.

4.4. Comparison of complexity between dGN and fast dGN. In general, the dGN algorithm [20,29] constructs the whole approximate Hessian of size $R T \times R T$ from its submatrices $\mathbf{H}^{(n, m)}$ (see Appendix $\mathbf{B}$ ) which are deduced from $\mathbf{C}^{(n)}$ and $\mathbf{\Gamma}^{(n)}$. Computation of $\mathbf{C}^{(n)}$ and $\Gamma^{(n)}$ are with of complexity $O\left(R^{2} T\right)$ and $O\left(N R^{2}\right)$, respectively. According to Theorem B.2, each off-diagonal submatrix has a complexity of $O\left(R^{2} I_{n} I_{m}\right)$, it follows that computation of the whole $\mathbf{H}$ has the complexity of $O\left(R^{2} T^{2}\right)$. Note that $\mathbf{H}$ has $R^{2} T^{2}$ elements. Inverse $\mathbf{H}^{-1}$ can be computed with a complexity of $O\left(R^{3} T^{3}\right)$. The gradient $g$ is computed at a cost of $O(N R J)$. Thus dGN has a complexity per iteration of $O\left(N R J+R^{3} T^{3}\right)$.

Complexity of the fLM algorithm is analyzed for each step in Algorithm 1 as follows Step 3 computes $N$ matrices $\mathbf{C}^{(n)}$ and $\boldsymbol{\Gamma}^{(n)}$ with complexity $O\left(R^{2} T\right)$ and $O\left(N R^{2}\right)$ as in dGN. Hence, building up $\mathbf{K}$ is of complexity $O\left(N(N-1)(N-2) R^{2}\right)=O\left(N^{3} R^{2}\right)$.

Step 4 inverts $\boldsymbol{\Gamma}_{\mu}^{(n)}, n=1,2, \ldots, N$ at a cost of $O\left(N R^{3}\right)$. 
Step 5 computes the damped factors $\mathbf{A}^{(n)}$ at a cost of $O(N R J)$, and is one of the most expensive steps in the fast dGN algorithm. We note that the large workload $\mathbf{Y}_{(n)} \bigodot_{k \neq n} \mathbf{A}^{(k)}$ is used for evaluation of gradient, and exists in all CP algorithms such as ALS, OPT.

Step 7 builds up the block diagonal matrix $\boldsymbol{\Psi}_{\mu}$ with a complexity $O\left(N R^{4}\right)$.

Step 8 solves the inverse problem $\boldsymbol{\Phi}^{-1} \boldsymbol{w}_{\mu}$ with a cost of $O\left(N^{3} R^{6}\right)$. This step is much faster than inverse of the approximate Hessian $O\left(R^{3} T^{3}\right)$ due to $R \ll I_{n}$ or $N R<T$.

Instead of construction of the approximate Hessian, the fLM algorithm builds up the much smaller matrix $\boldsymbol{\Phi}$ of size $N R^{2} \times N R^{2}$. Hence, besides the cost of computation of the gradient or the damped ALS factors, fLM computes $\boldsymbol{\Phi}$ and $\boldsymbol{\Phi}^{-1}$ at a cost of $O\left(R^{2} T+N^{3} R^{6}\right)$ which is much smaller than the cost for construction of $\mathbf{H}$ and for $\mathbf{H}^{-1}$ in dGN.

The total expense of fLM per one iteration is approximately $O\left(N R J+N^{3} R^{6}\right)$. For $N>7$, the proposed algorithm has the same order of complexity as that of ALS. However, fLM is much faster than ALS because it requires less iterations than ALS.

4.5. Damping parameter in the $\mathbf{L M}$ algorithm. The choice of damping parameter $\mu$ in the fast dGN algorithms (4.3) affects the direction and the step size $\Delta \mathfrak{a}=\mathbf{H}_{\mu}^{-1} \mathbf{g}$ in the update rule (3.3): $\mathfrak{a} \leftarrow \mathfrak{a}+\Delta \mathfrak{a}$ [18]. In this paper, the damping parameter $\mu$ is updated using the efficient strategy proposed by Nielsen [18]:

$$
\begin{aligned}
& \mu \leftarrow \begin{cases}2 \max \left\{\frac{1}{3}, 1-(2 \rho-1)^{3}\right\}, & \rho>0, \\
2 \mu, & \text { otherwise, }\end{cases} \\
& \rho=\frac{\left\|\boldsymbol{e}_{t-1}\right\|_{2}^{2}-\left\|\boldsymbol{e}_{t}\right\|_{2}^{2}}{\Delta \mathfrak{a}^{T}(\boldsymbol{g}+\mu \Delta \mathfrak{a})}, \\
& \boldsymbol{g}=\mathbf{J}^{T}(\boldsymbol{y}-\hat{\boldsymbol{y}})=\left[\begin{array}{c}
\operatorname{vec}\left(\mathbf{Y}_{(1)}\left(\bigodot_{k \neq 1} \mathbf{A}^{(k)}\right)-\mathbf{A}^{(1)} \boldsymbol{\Gamma}^{(1)}\right) \\
\vdots \\
\operatorname{vec}\left(\mathbf{Y}_{(N)}\left(\bigodot_{k \neq N} \mathbf{A}^{(k)}\right)-\mathbf{A}^{(N)} \boldsymbol{\Gamma}^{(N)}\right)
\end{array}\right] \in \mathbb{R}^{R T} .
\end{aligned}
$$

where $\boldsymbol{e}_{t}=\operatorname{vec}\left(\boldsymbol{y}-\hat{\boldsymbol{y}}_{t}\right)$, the gradient $\boldsymbol{g}$ can be straightforwardly derived as in (D.1) or in [29,31]. The factors $\mathbf{A}^{(n)}$ will be updated unless the new approximate is lower than the previous one: $\left\|\boldsymbol{e}_{t}\right\|_{2}<\left\|\boldsymbol{e}_{t-1}\right\|_{2}$. The algorithm should stop when $\mu$ increases to a sufficiently large value (e.g., $10^{30}$ ). In practice, the factors $\mathbf{A}^{(n)}$ are often initialized using the mode- $n$ singular vectors of the data tensor [5, 7, 14], then run over ALS (3.1) after few iterations. According to the CP model (2.3), all the components $\boldsymbol{a}_{r}^{(n)}(n \neq N)$ except ones of the last factor are unit-length vectors. The initial value of the damping parameter $\mu$ is chosen as the maximum diagonal entry of $\mathbf{H}$ as

$$
\begin{aligned}
\mu_{0} & =\tau \max \{\operatorname{diag}(\mathbf{H})\}=\tau \max \left\{\operatorname{diag}\left(\boldsymbol{\Gamma}^{(1)}\right) \cdots \operatorname{diag}\left(\boldsymbol{\Gamma}^{(n)}\right) \cdots \operatorname{diag}\left(\boldsymbol{\Gamma}^{(N)}\right)\right\} \\
& =\tau \max \left\{1, \operatorname{diag}\left(\mathbf{C}^{(N)}\right)\right\},
\end{aligned}
$$

where $\tau$ is typically in the range of $\left[10^{-8}, 1\right]$.

5. Complex-valued tensor factorization. This section aims to extend the dGN algorithms to complex-valued tensors. Although a real-valued tensor is considered as a complexvalued tensor with zero imaginary part, for simplicity algorithms for real- and complex-valued 
tensors are introduced in two separate sections. For the complex case, CP model is to find complex-valued factors $\mathbf{A}^{(n)} \in \mathbb{C}^{I_{n} \times R}$.

The damped Gauss-Newton-like update rule 3.3 is rewritten to update complex-valued factors [8, 23]

$$
\mathfrak{a} \leftarrow \mathfrak{a}+\left(\mathbf{J}^{H} \mathbf{J}+\mu \mathbf{I}\right)^{-1} \mathbf{J}^{H}(\mathfrak{Y}-\hat{\mathfrak{Y}})
$$

where symbol " $H$ " denotes the Hermitian transpose, and the Jacobian $\mathbf{J}$ is given in $(\mathrm{B} .1$. The approximate Hessian $\mathbf{H}=\mathbf{J}^{H} \mathbf{J}$ slightly changes from that for the real-valued tensors. A fast and efficient computation method for the complex-valued approximate Hessian $\mathbf{H}$ will be presented so that the final update rule does not employ both of the Jacobian and the approximate Hessian. We consider $\mathbf{H}$ as a partitioned matrix of $(N \times N)$ sub-matrices $\mathbf{H}^{(n, m)} \in \mathbb{C}^{R I_{n} \times R I_{m}}$, $n, m=1,2, \ldots, N$. Each sub-matrix $\mathbf{H}^{(n, m)}$ is a partitioned matrix of $(R \times R)$ subsub matrices $\mathbf{H}_{r, s}^{(n, m)} \in \mathbb{C}^{I_{n} \times I_{m}}, n, m=1,2, \ldots, N, r, s=1,2, \ldots, R$. The explicit expression of the approximate Hessian $\mathbf{H}$ is deduced from the following theorems which can be derived in a similar manner as for real valued tensors.

Theorem 5.1 (Subsub-matrices $\mathbf{H}_{r, s}^{(n, m)}$ ). $\mathbf{H}_{r, s}^{(n, m)}$ are diagonal or rank-one matrices given by

$$
\mathbf{H}_{r, s}^{(n, m)}=\delta_{n, m} \gamma_{r, s}^{(n)} \mathbf{I}_{I_{n}}+\left(1-\delta_{n, m}\right) \gamma_{r, s}^{(n, m)} \boldsymbol{a}_{r}^{(n)} \boldsymbol{a}_{s}^{(m) H}
$$

where $\gamma_{r, s}^{(n)}$ are the $(r, s)$ entries of the Hermitian matrices $\boldsymbol{\Gamma}^{(n, m)}=\underset{k \neq n, m}{\circledast} \mathbf{A}^{(k) H} \mathbf{A}^{(k)}$.

Theorem 5.2 (Sub-matrices $\mathbf{H}^{(n, m)}$ ). With $\mathbf{K}$ defined as in (4.2), $\mathbf{H}^{(n, m)}$ are expressed in an explicit form as

$$
\mathbf{H}^{(n, m)}=\delta_{n, m}\left(\boldsymbol{\Gamma}^{(n)} \otimes \mathbf{I}_{I_{n}}\right)+\left(\mathbf{I}_{R} \otimes \mathbf{A}^{(n)}\right) \mathbf{K}^{(n, m)}\left(\mathbf{I}_{R} \otimes \mathbf{A}^{(m) H}\right)
$$

Theorem 5.3 (Low-Rank Adjustment). For $N R \ll T$, the approximate Hessian $\mathbf{H}=\mathbf{J}^{H} \mathbf{J}$ can be expressed as a low-rank adjustment given by

$$
\mathbf{H}=\mathbf{G}+\mathbf{Z} \mathbf{K} \mathbf{Z}^{H},
$$

where sparse matrices $\mathbf{G}, \mathbf{Z}$ and $\mathbf{K}$ are defined as in (4.10), (4.11) and (4.2).

The damped Gauss-Newton algorithms for complex-valued tensor factorization are stated in following theorems:

THEOREM 5.4 (damped GN algorithm for complex-valued tensor factorizations). The factors $\mathbf{A}^{(n)}$ are updated using the rule given by

$$
\mathfrak{a} \leftarrow \mathfrak{a}+(\mathbf{H}+\mu \mathbf{I})^{-1} \mathbf{g},
$$

where the approximate Hessian $\mathbf{H}$ is defined in Theorems 5.1 or 5.2 an Levenberg-Marquardt regularization parameter $\mu>0$ and the gradient $\mathbf{g} \in \mathbb{C}^{R T}$ is computed as

$$
\boldsymbol{g}=\left[\operatorname{vec}\left(\mathbf{Y}_{(n)}\left(\bigodot_{k \neq n} \mathbf{A}^{(k) *}\right)-\mathbf{A}^{(n)} \boldsymbol{\Gamma}^{(n)^{T}}\right)^{T}\right]_{n=1}^{N}
$$

where symbol '*' denotes the complex conjugate.

Theorem 5.5 (fast dGN for low rank approximation). For $N R \ll T$, the factors $\mathbf{A}^{(n)}$ are updated using the fast update rule given by 


$$
\mathbf{A}^{(n)} \leftarrow \mathbf{A}_{\mu}^{(n)}+\mathbf{A}^{(n)}\left(\mathbf{I}_{R}-\left(\mathbf{F}_{n}+\mathbf{\Gamma}^{(n)^{T}}\right) \widetilde{\mathbf{\Gamma}}_{\mu}^{(n)}\right)
$$

where $\mathbf{F}_{n}$ are frontal slices of a 3-D tensor $\mathcal{F}$ whose $\operatorname{vec}(\mathcal{F})=\mathbf{B}_{\mu} \boldsymbol{w}_{\mu}, \mathbf{B}_{\mu}=\left(\mathbf{K}^{-1}+\boldsymbol{\Psi}_{\mu}\right)^{-1}$ if $\mathbf{K}$ is invertible, or $\mathbf{B}_{\mu}=\mathbf{K}\left(\mathbf{I}+\boldsymbol{\Psi}_{\mu} \mathbf{K}\right)^{-1}$, and $\boldsymbol{w}_{\mu}$ is computed from the damped ALS factors $\mathbf{A}_{\mu}^{(n)}$

$$
\begin{aligned}
\widetilde{\boldsymbol{\Gamma}}_{\mu}^{(n)} & =\left(\boldsymbol{\Gamma}^{(n)}+\mu \mathbf{I}_{R}\right)^{-1}, \\
\boldsymbol{\Psi}_{\mu} & =\operatorname{blkdiag}\left(\widetilde{\boldsymbol{\Gamma}}_{\mu}^{(n)} \otimes \mathbf{A}^{(n) H} \mathbf{A}^{(n)}\right)_{n=1}^{N}, \\
\boldsymbol{w}_{\mu} & =\operatorname{vec}\left(\left[\mathbf{A}^{(n) H} \mathbf{A}_{\mu}^{(n)}-\boldsymbol{\Gamma} \widetilde{\boldsymbol{\Gamma}}_{\mu}^{(n)}\right]_{n=1}^{N}\right), \\
\mathbf{A}_{\mu}^{(n)} & =\mathbf{Y}_{(n)}\left(\bigodot_{k \neq n} \mathbf{A}^{(k) *}\right) \widetilde{\boldsymbol{\Gamma}}_{\mu}^{(n)} .
\end{aligned}
$$

6. Experiments - Computer simulations. The $\mathrm{CP}$ algorithms were verified for difficult data with collinear factors in all modes (swamp). Collinearity degree of factors was controlled by mutual angles between their components. Collinear factors $\mathbf{A}^{(n)}$ were generated from random orthonormal factors $\mathbf{U}^{(n)}$

$$
\boldsymbol{a}_{r}^{(n)}=\boldsymbol{u}_{1}^{(n)}+v \boldsymbol{u}_{r}^{(n)}, \quad v \in(0,1], \forall n, \forall r \neq 1 .
$$

Mutual angles $\theta_{q, r}$ between $\boldsymbol{a}_{q}^{(n)}$ and $\boldsymbol{a}_{r}^{(n)}, q \neq r$ were in a range of $\left(0,60^{\circ}\right]$ for $v \in(0,1]$

$$
\tan \left(\theta_{q, r}\right)= \begin{cases}v, & q=1, \\ v \sqrt{v^{2}+2}, & q \neq 1, r .\end{cases}
$$

For example, $v=0.1,0.2, \ldots, 1$ yield $\theta_{1, r}=6^{\circ}, 11^{\circ}, 17^{\circ}, 22^{\circ}, 27^{\circ}, 31^{\circ}, 35^{\circ}, 39^{\circ}, 42^{\circ}, 45^{\circ}$, and $\theta_{q, r}=8^{\circ}, 16^{\circ}, 23^{\circ}, 30^{\circ}, 37^{\circ}, 43^{\circ}, 48^{\circ}, 52^{\circ}, 56^{\circ}, 60^{\circ}, q \neq 1, q \neq r$, respectively. For high $v$ such as $v=2, \theta_{1, r} \approx 63^{\circ}$ and $\theta_{q, r} \approx 78^{\circ}$, tensor can be quickly factorized by CP algorithms. The higher the parameter $v$, the lower the collinearity of factors. It is more difficult to factorize tensors with lower $v$ (e.g., $v=0.1,0.2$ ). However, when $v>3$, another issue arises from large difference in magnitude between components. The tensors are still difficult to factorize even thought collinearity of factors is low $\left(\theta_{1, r}>71^{\circ}\right)$. CP tensors, as in (2.3), can equivalently be constrained to be of the form

$$
\boldsymbol{y}=\sum_{r=1}^{R} \lambda_{r} \boldsymbol{a}_{r}^{(1)} \circ \boldsymbol{a}_{r}^{(2)} \circ \cdots \circ \boldsymbol{a}_{r}^{(N)}
$$

where $\left\|\boldsymbol{a}_{r}^{(n)}\right\|_{2}=1, \forall r$, and each $\lambda_{r}$ encodes the magnitude. For this experiment $\lambda_{1}=1$, and $\lambda_{r}=\left(1+v^{2}\right)^{N / 2}, \forall r>1$. Therefore, for $v=3,4,5$ and $N=3, \lambda_{r}=31.6,70.1,132.6, \forall r \neq 1$, respectively. That means the components $\boldsymbol{a}_{r}^{(n)}, r=2, \ldots, R$ are relatively larger than the first component. We analyze synthetic tensors for two cases: error-free and noisy data with additive white Gaussian noise at SNR $\left(=-10 \log _{10} \frac{\|\boldsymbol{y}\|_{F}^{2}}{\sigma^{2} \Pi I_{n}}\right)=30 \mathrm{~dB}$ or $40 \mathrm{~dB}$ added to the data tensor $\widetilde{\boldsymbol{y}}=\boldsymbol{y}+\sigma \mathcal{N}$, where $\mathcal{N}$ denotes a normally-distributed random tensor of zero mean and unit variance whose $n_{i_{1} i_{2} \ldots i_{N}} \sim N(0,1)$. 
In order to evaluate the factorizations for collinear data, we measured the Median Squared Angular Error (MedSAE) over multiple runs between the original and estimated components $\boldsymbol{a}_{r}^{(n)}, \widehat{\boldsymbol{a}}_{r}^{(n)}$ after matching their orders defined as

$$
\operatorname{MedSAE}\left(\boldsymbol{a}_{r}^{(n)}, \widehat{\boldsymbol{a}}_{r}^{(n)}\right)=10 \log _{10}\left(\operatorname{median}\left(\alpha_{r}^{(n) 2}\right)\right)(\mathrm{dB}),
$$

where $\alpha_{r}^{(n)}=\arccos \frac{\boldsymbol{a}_{r}^{(n) H} \widehat{\boldsymbol{a}}_{r}^{(n)}}{\left\|\boldsymbol{a}_{r}^{(n)}\right\|_{2}\left\|\widetilde{\boldsymbol{a}}_{r}^{(n)}\right\|_{2}}$. Cramér-Rao Induced Bound (CRIB) on $\alpha_{r}^{(n) 2}$ was computed from the Cramér-Rao lower bound (CRLB) for estimating the component $\boldsymbol{a}_{r}^{(n)}$ [15,25,-27]

$$
\operatorname{CRIB}\left(\alpha_{r}^{(n) 2}\right)=10 \log _{10} \frac{\operatorname{tr}\left(\left(\mathbf{I}_{I_{n}}-\boldsymbol{a}_{r}^{(n) T} \boldsymbol{a}_{r}^{(n)} /\left\|\boldsymbol{a}_{r}^{(n)}\right\|^{2}\right) \operatorname{CRLB}\left(\boldsymbol{a}_{r}^{(n)}\right)\right)}{\left\|\boldsymbol{a}_{r}^{(n)}\right\|^{2}} \quad(\mathrm{~dB}) .
$$

For our simulations, due to the same collinearity degree $v$ for all the components, we have

$$
\begin{array}{lll}
\operatorname{CRIB}\left(\alpha_{r}^{(n) 2}\right)=\operatorname{CRIB}\left(\alpha_{r}^{(1) 2}\right), & \forall r, \forall n, \\
\operatorname{CRIB}\left(\alpha_{r}^{(n) 2}\right)=\operatorname{CRIB}\left(\alpha_{2}^{(n) 2}\right), & \forall n, r=2, \ldots, R .
\end{array}
$$

The average MedSAEs for the estimated components were compared against the average CRIB. It is important to note that an MedSAE lower than $-30 \mathrm{~dB},-26 \mathrm{~dB}$ or $-20 \mathrm{~dB}$ means two components are different by a mutual angle less than $2^{\circ}, 3^{\circ}$ and $6^{\circ}$, respectively. Practical simulations show that it is difficult for MedSAE to reach a CRIB $\geq-30 \mathrm{~dB}$, since collinearity of factors has been destroyed by noise. Discussion on effects of noise on collinear data in Appendix $\mathrm{F}$ gives us insight into when $\mathrm{CP}$ algorithms are not stable, and when they succeed in retrieving collinear factors from noisy tensors.

6.1. Comparison between dGN and fLM for 3-D tensor factorizations. This section compares performance of fLM and the standard dGN algorithm in the Matlab routines PARAFAC3W developed by Tomasi [28, 32]. The dGN algorithm [28] computes the approximate Hessian and gradient, and employs Cholesky decomposition and back substitution to solve the inverse problems $\mathbf{H}^{-1} \boldsymbol{g}$. Unfortunately, this toolkit supports only 3-D data. The $\mathrm{fLM}_{\mathrm{a}}$ algorithm was verified, and shortly denoted by fLM.

In the first set of experiments, random synthetic tensors were generated from 3 collinear factor matrices of size $I \times R$ where $I=100$ and $R=5,10,20,30,40,60$ and $v=0.5$. From each noise-free CP tensor $\boldsymbol{y}$ composed from $\mathbf{A}^{(n)} \in \mathbb{R}^{I \times R}$, twenty noisy tensors $\widehat{\boldsymbol{y}}$ of $30 \mathrm{~dB}$ SNR were generated. There are in total 200 rank- $R$ tensors $\widehat{y}$. MedSAE for each component was deduced from 200 runs for each test case.

Both algorithms were initialized by the same factors which were the mode- $n$ singular vectors of the data tensor [7]. Algorithms stop when 10 differences of successive relative errors $\varepsilon=\frac{\|y-\widehat{y}\|_{F}}{\|y\|_{F}}$ were lower than $10^{-8}$, or until the maximum number of iterations (1000) was achieved. Execution time for each algorithm was measured using the stopwatch command: "tic" "toc" of MATLAB release 2009a on a computer which had 2 quadcore $3.33 \mathrm{GHz}$ processors and 64 GB memory. Tucker compression was not used in the simulations. The $\mathrm{dGN}$ in [28] was adapted to follow the same stopping criteria and the same computational time measurements, while its other parameters were set to default values.

Fig. 6.1(a) visualizes the overall execution times in seconds and the average execution times per iteration for both algorithms. The speed-up ratios for the overall decomposition between dGN and fLM were approximately 6.4, 14.6, 35.1, 16.7, 7.8 and 2.8 times for $R=5$, $10,20,30,40,60$ respectively, while the speed-up ratios per iteration were respectively 5.6, $14.7,20,7,11.3,6.5$ and 2.7. We note that the numbers of iterations of dGN and fLM were slightly different because of differences between them in controlling the damping parameters. 


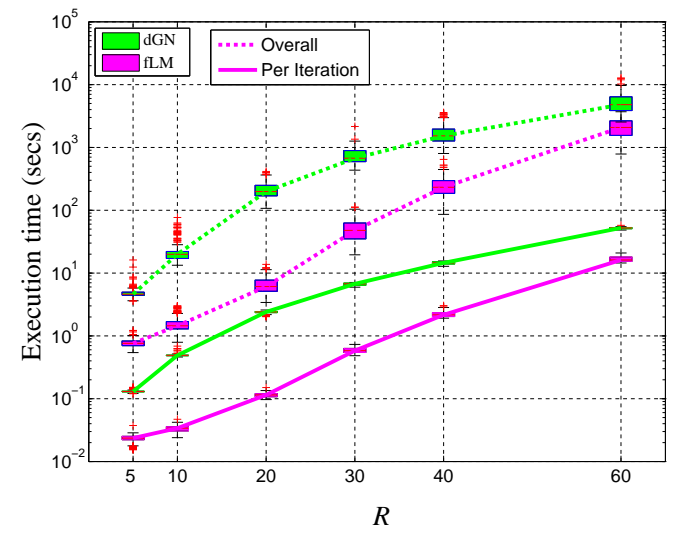

(a) Overall execution time and average execution time per iteration.

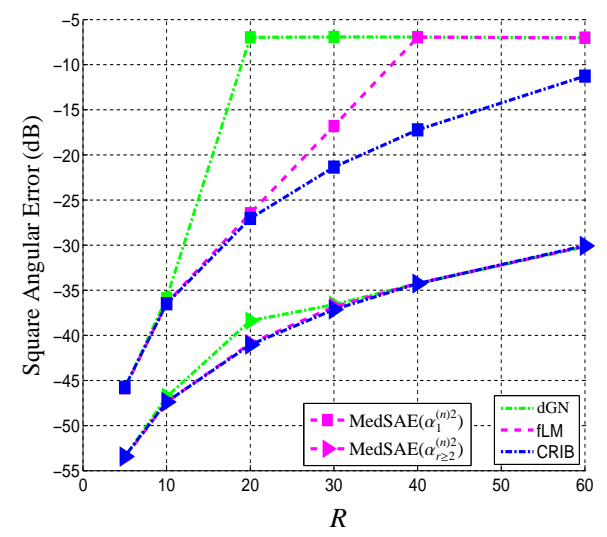

(b) MedSAE and CRIB

Fig. 6.1. Comparison between the $d G N$ (green lines) and fLM (magenta lines) algorithms for factorization of $100 \times 100 \times 100$ dimensional tensors composed by collinear factors for various $R$ at $S N R=30 \mathrm{~dB}:(a)$ the overall execution times in second (dashed lines) and the average execution times per iteration (solid lines); (b) the average MedSAE values $(d B)$ of the first components $\boldsymbol{a}_{1}^{(n)}$ (square marker) and of other components $\boldsymbol{a}_{r}^{(n)}$ (triangular marker), $r=2, \ldots, R, n=1,2,3$.

In Fig. 6.1(b) we illustrate the average MedSAE values of dGN [28] and fLM. The mean MedSAEs for the first components $\boldsymbol{a}_{1}^{(n)}, n=1, \ldots, N$ were calculated over $N \operatorname{MedSAE}\left(\alpha_{1}^{(n) 2}\right)$; whereas the mean MedSAEs for the other components $\boldsymbol{a}_{r}^{(n)}, r=2,3, \ldots, R, n=1, \ldots, N$ were calculated over $(N \times(R-1)) \operatorname{MedSAE}\left(\alpha_{r \geq 2}^{(n) 2}\right)$. Fig 6.1(b) shows that the average values of $\operatorname{MedSAE}\left(\alpha_{r}^{(n) 2}\right), r \geq 2, \forall n$, asymptotically attained the CRIB. It means that both dGN and fLM well reconstructed components $\boldsymbol{a}_{r}^{(n)}, r=2, \ldots, R, \forall n$ even for $R=60$. To be accurate, CRIB is a theoretical lower bound on the mean of the square angular error, not on the median. In these simulations, the median and mean SAEs appeared to be nearly identical so that only the former one is shown.

For the first components $\boldsymbol{a}_{1}^{(n)}$, performances of dGN and fLM were equivalent in the sense of collinearity reconstruction for small $R=5,10$. For $R=20,30$, fLM still reconstructed the first components. Note that although MedSAEs were different, the relative approximation errors $\varepsilon$ of two algorithms were almost the same but they were not presented here. The difference in component reconstruction was caused by implementation of the control strategy for damping parameter. For $R \geq 40$, the average MedSAEs of the two algorithms were much worse than the CRIB, and they were not able to reconstruct the first components. Indeed, we cannot recover the first components due to noise for high $R$.

In order to analyze complexity of the two algorithms for higher ranks $R \rightarrow I$, we decomposed tensors of the same dimensions whose entries were randomly generated. The rank $R$ varied from 5 to $I=100$. The amount of allocated memory and average execution time per iteration were measured on the computer (PC1) in the previous simulations and on a computer (PC2) which had $2.67 \mathrm{GHz}$ i7 CPU and $4 \mathrm{~GB}$ of memory. The results were summarized in Fig. 6.2. For high rank $R \geq 50$, dGN required more than 4 GB of memory and could consume 20 GB of memory for $R=100$ whereas fLM need less than 4 GB of memory. On PC1 which had 64 GB of memory, fLM was slightly more time consuming for $R \geq 90$ than $\mathrm{dGN}$ because the advantage of the fast inversion in (4.6) was lost. However, dGN became dramatically time consuming on PC2 when $R \geq 40$. 


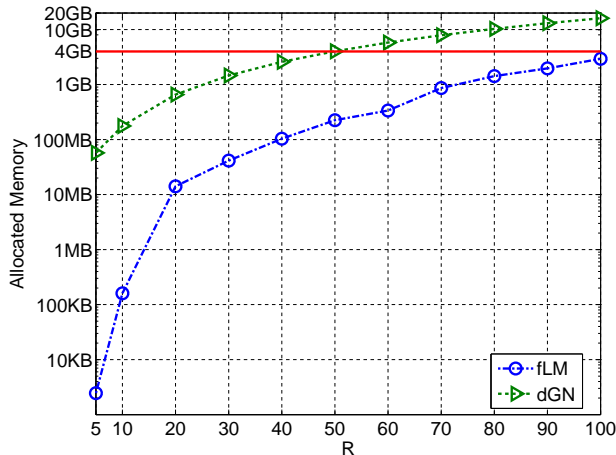

(a) Allocated memory.

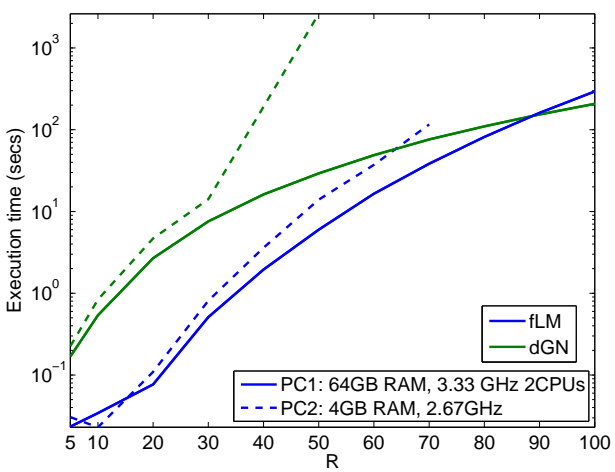

(b) Execution time per iteration.

FIG. 6.2. Memory requirements and execution time per iteration of $d G N$ and $f L M$ in approximation of $100 \times$ $100 \times 100$ dimensional tensors by rank-R tensors where $R=5,10,20, \ldots, 100$.

6.2. Factorization of higher-order real-valued tensors. The proposed algorithms have been extensively verified and compared with the ALS algorithm plus line seach in the N-way toolbox [2], for 4-D tensors of size $I_{n}=50$, various ranks $R=5,10,15$, and with different collinearity degree $v=0.1,0.3,0.5,0.7,0.9$. The 4 -D tensors were corrupted by additive Gaussian noise at SNR $=40 \mathrm{~dB}$. For each pair $(v, R)$ MedSAE was computed from 400 runs. Execution times (seconds) were measured on a computer that had 6-core i7 $3.33 \mathrm{GHz}$ processor and 24 GB memory.

Algorithms were analyzed under the same experimental conditions as in the previous simulations. They iterated until successive relative errors $\varepsilon$ were lower than $10^{-12}$, or the maximum number of iterations (5000) was achieved. The ALS algorithm plus line seach (ALSls) was adapted to have the same stopping criteria.

At $\mathrm{SNR}=40 \mathrm{~dB}$ and ranks $R=5,10,15, \mathrm{CRIBs}$ are relatively high $(>40 \mathrm{~dB})$ for most $v$ (see Fig.6.3(d). Hence, CPD algorithms easily estimated collinear factors and obtained high MedSAE comparable to the CRIB. Fig. 6.3(d) shows that MedSAEs of ALSls and fLM were almost similar and approached CRIB except those for $R=15$ and $v=0.1$. It should be noted that factorization became more difficult in the case of higher rank $R$ and lower $v$. Execution times of algorithms for different $R$ and $v$ are illustrated in Figs. 6.3(a) 6.3(c) The results indicate that the higher the collinearity degree (i.e., smaller $v$ ) the more time-consuming the algorithms. For example, ALSls on average ran 2083 iterations in 957 seconds to factorize 4-D noisy tensors when $R=10$ and $v=0.1$. However, when keeping the tensor size and rank $R$ and changing $v=0.9$, this algorithm ran 34 iterations in 14 seconds. For the same tensors with $v=0.1$, fLM took only 48.6 seconds on average to execute 384 iterations, and took 6 seconds for 21 iterations with $v=0.9$. That means fLM was 21 times faster than ALS with $v=0.1$. For 4-D tensors of $R=15$ and with $v=0.1$, ALSls ran 4225 iterations in 2255 seconds on average, while fLM took only 103 seconds to execute 494 iterations. Hence, fLM was 24.7 times faster than ALSls for the difficult test case. More execution times and speed ratios are given in Table 6.1. Speed ratio between ALSls and fLM was high for highly collinear data (e.g., $v=0,1$ ). For example, fLM was at least 17.1 times and up to 24.8 times faster than ALSls for collinear data with $v=0.1$. For lower collinearity degree, ALSls quickly factorized the tensor after few iterations. Although the speed ratio decreased, fLM was still approximately 3 times faster than ALSls. 


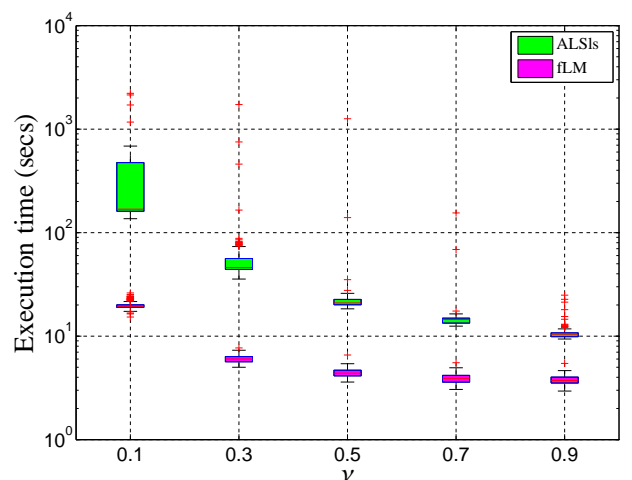

(a) 4-D tensors, $\mathbf{A}^{(n)} \in \mathbb{R}^{50 \times 5}, \mathrm{SNR}=40 \mathrm{~dB}$.

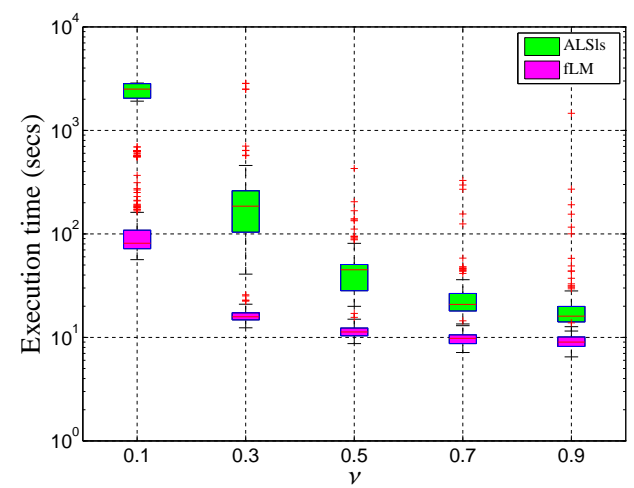

(c) 4-D tensors, $\mathbf{A}^{(n)} \in \mathbb{R}^{50 \times 15}, \mathrm{SNR}=40 \mathrm{~dB}$.

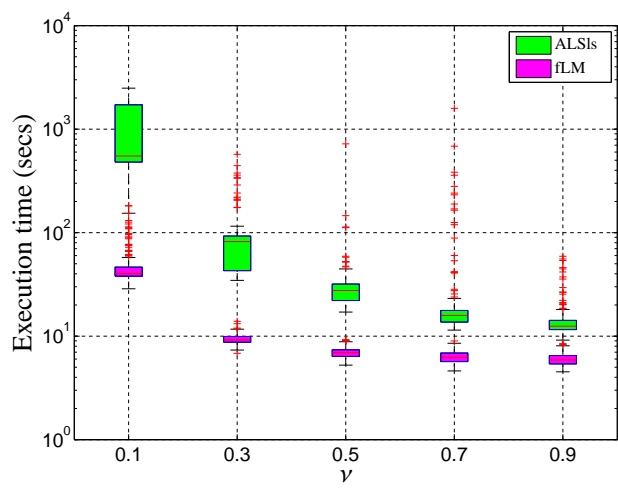

(b) 4-D tensors, $\mathbf{A}^{(n)} \in \mathbb{R}^{50 \times 10}, \mathrm{SNR}=40 \mathrm{~dB}$.

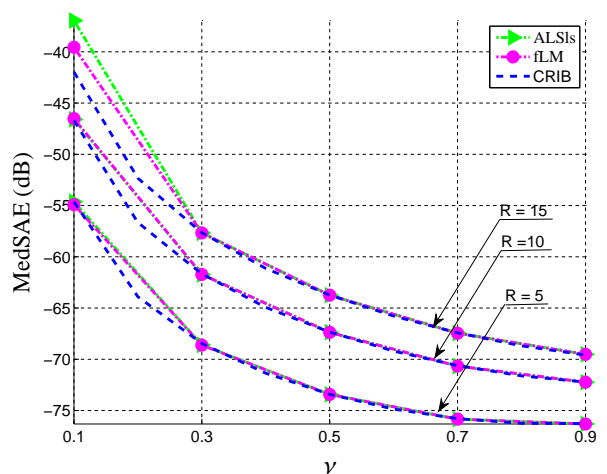

(d) 4-D tensors, $R=5,10,15, \mathrm{SNR}=40 \mathrm{~dB}$.

FIG. 6.3. Comparison between ALSls and fLM for factorizations of 4-D tensors of size $50 \times 50 \times 50 \times 50$ at $S N R=40 \mathrm{~dB}$. (a) (c) execution times (seconds) were measured when algorithms factorized tensors of various ranks $R=5,10,15$. (d) the average MedSAE (dB) for all components compared with CRIB.

6.3. Factorization of complex-valued tensors. In the next set of simulations, we considered factorization of complex-valued tensors. Factors $\mathbf{A}^{(n)} \in \mathbb{C}^{70 \times R}$ were generated in the same manner as for experiments in the previous section. However, they had random real and imaginary parts. In addition to collinearity degrees $v=0.1,0.2, \ldots, 0.5$, we considered $v=3,4,5$. We note that although collinearity of factors is low for high $v=3,4,5\left(\theta_{1, r}>71^{\circ}\right)$, the tensors are still difficult to factorize.

We compared fLM with ALS plus line search (ALSls). Algorithms stopped when differences between successive relative errors were lower than $10^{-8}$, or the maximum number of iterations (2000) was achieved. In Figs. 6.4(a) (b), we illustrate the average MedSAE of all factors for $70 \times 70 \times 70 \times 70$ dimensional tensors with ranks $R=5$ and 15 over 200 runs. ALSls achieved good performance with $v=0.2$, and excellent MedSAE with $v=0.3,0.4$ and 0.5. However, for high collinearity degree $v=4$ and 5, ALSls did not obtain perfect reconstruction. The fLM algorithm outperformed ALSls for all test cases. Figs. 6.4(c) (d) indicate that the number of iterations of ALSls tended to decrease gradually as $v$ increased from 0.1 to 5. For $v=3,4,5$, ALSls stopped after tens of iterations because there was not any significant change in the relative error. Figs. 6.4(c) (d) also reveal that fLM required less iterations for higher $v$. Difference in magnitude between components did not affect fLM. 
TABLE 6.1

Comparison of average execution times (seconds) between fLM and ALSls for factorizations of 4-D and 5-D tensors of size $I_{n}=50$ at $S N R=40 \mathrm{~dB}$ composed by collinear factors with various $v=0.1,0.3,0.5,0.9$ and for various $R$. For each pair $\left(N, I_{m}, R, v\right)$, speed-up ratio and execution times are given as indicated in the subtable at the bottom.

\begin{tabular}{|c|c|c|c|c|c|c|c|c|c|c|}
\hline \multirow{2}{*}{$\begin{array}{l}\text { Tensor's size } \\
\left(N-\mathrm{D}, I_{m} \times R\right)\end{array}$} & \multicolumn{10}{|c|}{ Collinear degree $v$} \\
\hline & \multicolumn{2}{|c|}{0.1} & \multicolumn{2}{|c|}{0.3} & \multicolumn{2}{|c|}{0.5} & \multicolumn{2}{|c|}{0.7} & \multicolumn{2}{|c|}{0.9} \\
\hline \multirow{2}{*}{$4-\mathrm{D}, 50 \times 5$} & 171 & 347 & 111 & 65 & 6 & 28 & 39 & 15 & 28 & 11 \\
\hline & 17.1 & 20 & 11.1 & 6 & 0 & 4.4 & 3.9 & 3.9 & 2.8 & 3.8 \\
\hline \multirow{2}{*}{$4-\mathrm{D}, 50 \times 10$} & 21.2 & 957 & 96 & 90 & & 34 & & 40 & & 11 \\
\hline & 21.2 & 49 & 9.0 & 9 & 4.9 & 7 & 6 & 6 & 2.5 & 6 \\
\hline \multirow{2}{*}{$4-D, 50 \times 15$} & \multirow{2}{*}{24.8} & 2,201 & & 263 & 42 & 48 & 3 & 29 & 29 & 29 \\
\hline & & 99 & 15.4 & 16 & 4.2 & 11 & 3 & 10 & 2.9 & 9 \\
\hline \multirow{2}{*}{$5-\mathrm{D}, 50 \times 5$} & \multirow{2}{*}{22} & 17,245 & & 2,747 & & 1,240 & & 821 & & 730 \\
\hline & & 790 & 8.1 & 346 & 4.6 & 453 & 4.2 & 205 & 3.4 & 251 \\
\hline
\end{tabular}

ratio $\frac{\text { Execution time }_{\mathrm{ALSls}} \text { (seconds) }}{\text { Execution time }_{\mathrm{fLM}} \text { (seconds) }}$

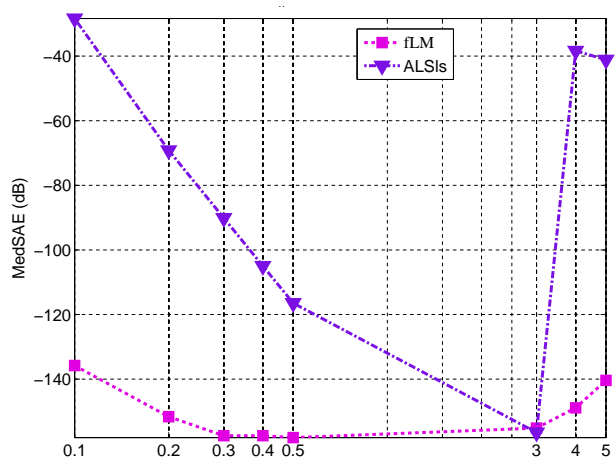

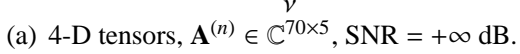

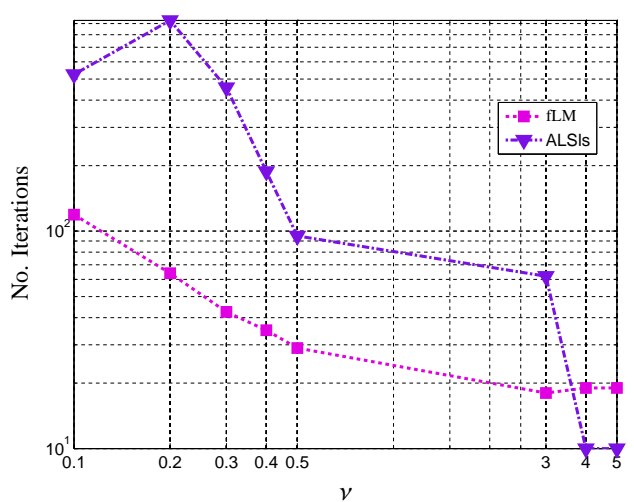

(c) 4-D tensors, $\mathbf{A}^{(n)} \in \mathbb{C}^{70 \times 5}$.

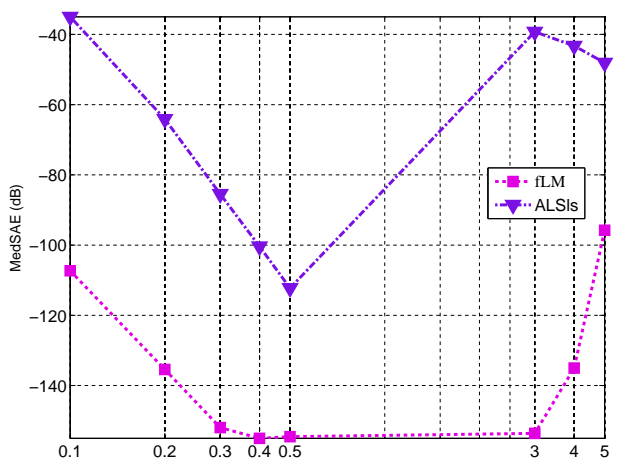

(b) 4-D tensors, $\mathbf{A}^{(n)} \in \stackrel{v}{\mathbb{C}^{70 \times 15}}, \mathrm{SNR}=+\infty \mathrm{dB}$.

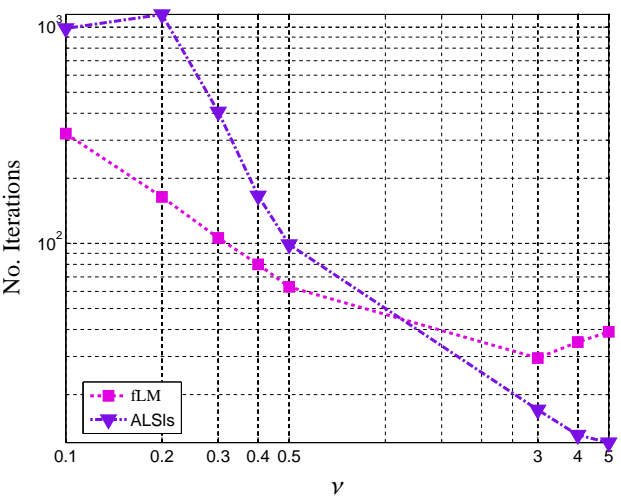

(d) 4-D tensors, $\mathbf{A}^{(n)} \in \mathbb{C}^{70 \times 15}$.

Fig. 6.4. Illustration for MSAE for factorization of 4-D complex-valued tensors with size $I_{n}=70$ and ranks $R=5$, 15. Algorithms stopped as they reached a derivative of successive relative errors of $10^{-8}$ or 2000 iterations.

7. Conclusions. Simulations for real- and complex-valued tensors confirmed the fLM algorithm was faster than dGN and ALS, and outperformed ALS in the sense of approxima- 
tion accuracy (MedSAE) for difficult test cases. Moreover, MedSAE of fLM was comparable to CRIB for most test cases even for noisy tensors. For the collinearity modification used in the simulations, we also show that for the same tensor size and collinearity degree, the higher rank $R$ the data tensor has, the more difficult the factorization is to retrieve the factor. For the same size $I_{n}$, rank $R$, and collinearity degree, the higher the dimensions of the data tensor, the higher the performance of factorization can be achieved.

Most CP algorithms incorporated with line-search techniques work well for general data, but often fail for highly collinear data with bottlenecks or swamps. The dGN/LM algorithms [20,29] can deal with such data, but demand extreme computational cost associated with large-scale inverse of approximate Hessians. In this paper, by employing the special structure of the approximate Hessian, a fast inverse for the approximate Hessian has been derived, and low complexity damped Gauss Newton algorithms have been proposed for factorization of low rank real- and complex-valued tensors. The proposed algorithm avoids building up the whole approximate Hessian and its inverse by working with much smaller matrices of size $N R^{2} \times N R^{2}$ instead of $(R T \times R T)$. Extensive experiments for tensor factorizations showed that our algorithms outperformed "state-of-the-art" algorithms for difficult benchmarks for both real and complex-valued tensors. The proposed dGN/LM algorithms can be extended to the nonnegative CPD in which factors are nonnegative matrices. Moreover, our algorithms can be simplified to estimate only one factor for supersymmetric tensor factorization which can be found in multiway clustering, or to the INDSCAL decomposition [5, 15].

Acknowledgments. The authors wish to thank the referees for the very constructive and detailed comments and suggestions which led to major improvements in the manuscript. They also thank for Dr. Benedikt Lösch and Mr. Austin Brockmeier for their suggestions that helped improving the manuscript.

Appendix A. Commutation Matrices. A commutation matrix $\mathbf{Q}_{n}$ expresses connection between vectorizations of tensor unfoldings, and often exists in construction of the Jacobian $\mathbf{J}$ and the approximate Hessian $\mathbf{H}$ in dGN algorithms for CP and Tucker decompositions [22].

LEMMA A.1. (mode- $n$ to mode-1 unfolding) Commutation matrix $\mathbf{Q}_{n}$ which maps $\operatorname{vec}\left(\mathbf{A}_{(1)}\right)=$ $\operatorname{vec}(\mathcal{A})=\mathbf{Q}_{n} \operatorname{vec}\left(\mathbf{A}_{(n)}\right)$ is given by $\mathbf{Q}_{n}=\mathbf{I}_{I_{n+1: N}} \otimes \mathbf{P}_{I_{1: n-1}, I_{n}}$, with $I_{i: j}=\prod_{k=i}^{j} I_{k}$.

Appendix B. Proof of Theorem 4.2

In order to prove Theorem 4.2, we seek explicit expressions for the Jacobian and the approximate Hessian in the next section.

LEMMA B.1. The Jacobian matrix $\mathbf{J}$ has a form of [20, 31]

$$
\mathbf{J}=\left[\mathbf{Q}_{n}\left(\left(\bigodot_{k \neq n} \mathbf{A}^{(k)}\right) \otimes \mathbf{I}_{I_{n}}\right)\right]_{n=1}^{N} .
$$

We express the approximate Hessian $\mathbf{H}$ as an $N \times N$ block matrix $\mathbf{H}=\left[\mathbf{H}^{(n, m)}\right]_{n, m}, \mathbf{H}^{(n, m)}$ of size $R I_{n} \times R I_{m}$.

Theorem B.2. (see also [20, 29]) A submatrix $\mathbf{H}^{(n, m)}$ has an explicit expression given by

$$
\mathbf{H}^{(n, m)}=\delta_{n, m}\left(\boldsymbol{\Gamma}^{(n)} \otimes \mathbf{I}_{I_{n}}\right)+\left(\mathbf{I}_{R} \otimes \mathbf{A}^{(n)}\right) \mathbf{K}^{(n, m)}\left(\mathbf{I}_{R} \otimes \mathbf{A}^{(m) T}\right), \quad \forall n, \forall m .
$$

By establishing expressions for submatrices $\mathbf{H}^{(n, m)}$, we can prove Theorem 4.2 .

Proof. (Theorem 4.2) From (B.2), we construct a sparse matrix G consisting all block matrices $\mathbf{H}^{(n)} n=1,2, \ldots, N$, that is

$$
\mathbf{G}=\operatorname{blkdiag}\left(\mathbf{H}^{(n)}\right)_{n=1}^{N}=\operatorname{blkdiag}\left(\boldsymbol{\Gamma}^{(n)} \otimes \mathbf{I}_{I_{n}}\right)_{n=1}^{N} .
$$


From Theorem B.2, and by using the product of block matrices, it is straightforward to decompose $\mathbf{H}-\mathbf{G}$ into three matrices defined in Theorem 4.2 as

$$
\mathbf{H}-\mathbf{G}=\mathbf{Z} \mathbf{K} \mathbf{Z}^{T} .
$$

$\square$

\section{Appendix C. Proof of Theorem 4.3 .}

Proof. The damped approximate Hessian $\mathbf{H}_{\mu}=\mathbf{G}+\mu \mathbf{I}_{R T}+\mathbf{Z K Z} \mathbf{Z}^{T}$ is adjusted from $\mathbf{G}_{\mu}=\mathbf{G}+\mu \mathbf{I}_{R T}$ by a low-rank matrix $\mathbf{Z K} \mathbf{Z}^{T}$. Hence, its inverse can be quickly computed by applying the binomial inverse theorem (see page 18 [13])

$$
\mathbf{H}_{\mu}^{-1}= \begin{cases}\mathbf{G}_{\mu}^{-1}-\mathbf{G}_{\mu}^{-1} \mathbf{Z}\left(\mathbf{K}^{-1}+\mathbf{Z}^{T} \mathbf{G}_{\mu}^{-1} \mathbf{Z}\right)^{-1} \mathbf{Z}^{T} \mathbf{G}_{\mu}^{-1}, & \text { if } \mathbf{K} \text { is invertible, } \\ \mathbf{G}_{\mu}^{-1}-\mathbf{G}_{\mu}^{-1} \mathbf{Z} \mathbf{K}\left(\mathbf{I}_{N R^{2}}+\mathbf{Z}^{T} \mathbf{G}_{\mu}^{-1} \mathbf{Z K}\right)^{-1} \mathbf{Z}^{T} \mathbf{G}_{\mu}^{-1}, & \text { otherwise. }\end{cases}
$$

Denote by $\widetilde{\mathbf{G}}_{\mu}$ inverse of the block diagonal matrix $\mathbf{G}_{\mu}$ which is also a block diagonal matrix

$$
\widetilde{\mathbf{G}}_{\mu}=\left(\operatorname{blkdiag}\left(\left(\boldsymbol{\Gamma}^{(n)}+\mu \mathbf{I}_{R}\right) \otimes \mathbf{I}_{I_{n}}\right)_{n=1}^{N}\right)^{-1}=\operatorname{blkdiag}\left(\widetilde{\boldsymbol{\Gamma}}_{\mu}^{(n)} \otimes \mathbf{I}_{I_{n}}\right)_{n=1}^{N} .
$$

Similarly, we denote $\mathbf{L}_{\mu}=\mathbf{G}_{\mu}^{-1} \mathbf{Z}$ and $\boldsymbol{\Psi}_{\mu}=\mathbf{Z}^{T} \mathbf{G}_{\mu}^{-1} \mathbf{Z}$. From (4.11) and by taking into ac$\operatorname{count}\left(\widetilde{\boldsymbol{\Gamma}}_{\mu}^{(n)} \otimes \mathbf{I}_{I_{n}}\right)\left(\mathbf{I}_{R} \otimes \mathbf{A}^{(n)}\right)=\widetilde{\boldsymbol{\Gamma}}_{\mu}^{(n)} \otimes \mathbf{A}^{(n)}$, we have

$$
\begin{aligned}
\mathbf{L}_{\mu} & =\mathbf{G}_{\mu}^{-1} \mathbf{Z}=\operatorname{blkdiag}\left(\widetilde{\boldsymbol{\Gamma}}_{\mu}^{(n)} \otimes \mathbf{I}_{I_{n}}\right)_{n=1}^{N} \operatorname{blkdiag}\left(\mathbf{I}_{R} \otimes \mathbf{A}^{(n)}\right)_{n=1}^{N} \\
& =\operatorname{blkdiag}\left(\widetilde{\boldsymbol{\Gamma}}_{\mu}^{(n)} \otimes \mathbf{A}^{(n)}\right)_{n=1}^{N} . \\
\boldsymbol{\Psi}_{\mu} & =\operatorname{blkdiag}\left(\widetilde{\boldsymbol{\Gamma}}_{\mu}^{(n)} \otimes \mathbf{C}^{(n)}\right)_{n=1}^{N} .
\end{aligned}
$$

Finally, we define $\mathbf{B}$ as in (4.6), and easily deduce (4.12) from (C.1).

\section{Appendix D. Proof of Lemma 4.5 ,}

Proof. From (B.1), (4.13), and note that $\operatorname{vec}(\mathcal{E})=\mathbf{Q}_{n} \operatorname{vec}\left(\mathcal{E}_{(n)}\right)$, where $\mathbf{Q}_{n}$ is defined in Lemma A.1 the product $\widetilde{\mathbf{G}}_{\mu} \boldsymbol{g}$ can be expressed in a block form as

$$
\begin{aligned}
\left(\widetilde{\mathbf{G}}_{\mu} \mathbf{J}^{T} \operatorname{vec}(\boldsymbol{E})\right)^{T} & =\left[\operatorname{vec}(\mathcal{E})^{T} \mathbf{Q}_{n}\left(\left(\left(\bigodot_{k \neq n} \mathbf{A}^{(k)}\right) \widetilde{\boldsymbol{\Gamma}}_{\mu}^{(n)}\right) \otimes \mathbf{I}_{I_{n}}\right)\right]_{n=1}^{N}=\left[\operatorname{vec}\left(\mathbf{E}_{(n)}\left(\bigodot_{k \neq n} \mathbf{A}^{(k)}\right) \widetilde{\boldsymbol{\Gamma}}_{\mu}^{(n)}\right)^{T}\right]_{n=1}^{N} \\
& =\left[\operatorname{vec}\left(\mathbf{Y}_{(n)}\left(\bigodot_{k \neq n} \mathbf{A}^{(k)}\right) \widetilde{\boldsymbol{\Gamma}}_{\mu}^{(n)}-\mathbf{A}^{(n)}\left(\bigodot_{k \neq n} \mathbf{A}^{(k)}\right)^{T}\left(\bigodot_{k \neq n} \mathbf{A}^{(k)}\right) \widetilde{\boldsymbol{\Gamma}}_{\mu}^{(n)}\right)^{T}\right]_{n=1}^{N} \\
& =\left[\operatorname{vec}\left(\mathbf{A}_{\mu}^{(n)}-\mathbf{A}^{(n)} \boldsymbol{\Gamma}^{(n)} \widetilde{\boldsymbol{\Gamma}}_{\mu}^{(n)}\right)^{T}\right]_{n=1}^{N} .
\end{aligned}
$$

Similarly, a convenient formula to compute $\mathbf{L}_{\mu}^{T} \boldsymbol{g}$ is given by

$$
\begin{aligned}
\boldsymbol{w}_{\mu} & =\mathbf{L}_{\mu}^{T} \mathbf{J}^{T} \operatorname{vec}(\boldsymbol{E})=\left[\operatorname{vec}\left(\mathbf{A}^{(n) T}\left(\mathbf{A}_{\mu}^{(n)}-\mathbf{A}^{(n)} \boldsymbol{\Gamma}^{(n)} \widetilde{\boldsymbol{\Gamma}}_{\mu}^{(n)}\right)\right)^{T}\right]_{n=1}^{N T} \\
& =\operatorname{vec}\left(\left[\mathbf{A}^{(n) T} \mathbf{A}_{\mu}^{(n)}-\boldsymbol{\Gamma} \widetilde{\boldsymbol{\Gamma}}_{\mu}^{(n)}\right]_{n=1}^{N}\right) .
\end{aligned}
$$


Finally, for each frontal slice $\mathbf{F}_{n}$ of the tensor $\mathcal{F} \in \mathbb{R}^{R \times R \times N}$ whose vec $(\mathcal{F})=\mathbf{B}_{\mu} \boldsymbol{w}_{\mu}$, we have

$$
\left(\widetilde{\boldsymbol{\Gamma}}_{\mu}^{(n)} \otimes \mathbf{A}^{(n)}\right) \operatorname{vec}\left(\mathbf{F}_{n}\right)=\operatorname{vec}\left(\mathbf{A}^{(n)} \mathbf{F}_{n} \widetilde{\boldsymbol{\Gamma}}_{\mu}^{(n)}\right) .
$$

From (4.14), we obtain (4.21). Each product inside (D.3) has a complexity of $O\left(I_{n} R^{2}+R^{3}\right)$. Hence, $\mathbf{L}_{\mu} \boldsymbol{f}$ in (4.21) has a complexity of $O\left(T R^{2}+N R^{3}\right) \approx O\left(T R^{2}\right)$ which is lower than $O\left(T R^{3}\right)$ by a factor $R$ for building up $\mathbf{L}_{\mu}$ and direct computation $\mathbf{L}_{\mu} f$. Furthermore, this fast computation does not use any significant temporary extra-storage. $\mathrm{Q}$

\section{Appendix E. Inverse of The Kernel Matrix K.}

THEOREM E.1. Inverse of $\mathbf{K}$ defined in (4.2) is a partitioned matrix $\widetilde{\mathbf{K}}=\mathbf{K}^{-1}$ whose blocks $\widetilde{\mathbf{K}}^{(n, m)}$, for $n=1, \ldots, N, m=1, \ldots, N$ are given by

$$
\widetilde{\mathbf{K}}^{(n, m)}=\left(\frac{1}{N-1}-\delta_{n, m}\right) \operatorname{diag}\left(\operatorname{vec}\left(\mathbf{C}^{(n)} \circledast \mathbf{C}^{(m)} \oslash \boldsymbol{\Gamma}\right)\right) \mathbf{P}_{R} .
$$

\section{Appendix F. Effects of noise on collinear data.}

This section discusses briefly effects of noise on factorization of collinear tensor generated by the modification 6.1). Consider matrix factorization of the mode- $n$ tensor unfolding

$$
\mathbf{Y}_{(n)}=\mathbf{A}^{(n)}\left(\bigodot_{k \neq n} \mathbf{A}^{(k)}\right)^{T}+\mathbf{E}_{(n)}
$$

Analysis of singular values of $\mathbf{Y}_{(n)}$ or eigenvalues of $\mathbf{Y}_{(n)} \mathbf{Y}_{(n)}^{T}$ allow predicting whether factorization succeeds in retrieving collinear factors from noisy tensors. This also gives insight into when $\mathrm{CP}$ algorithms are not stable, and yield non-unique solution.

The modification (6.1) can be expressed as $\mathbf{A}^{(n)}=\mathbf{U}^{(n)} \mathbf{Q}$, where $\mathbf{Q}=\left[\begin{array}{cc}1 & \mathbf{1}_{R-1}^{T} \\ \mathbf{0}_{R-1} & v \mathbf{I}_{R-1}\end{array}\right] \epsilon$ $\mathbb{R}^{R \times R}$. In theory, for noisy tensors $\boldsymbol{y}$ with $I_{n}=I, \forall n$, we have

$$
\mathbf{Y}_{(n)} \mathbf{Y}_{(n)}^{T}=\mathbf{A}^{(n)} \Gamma^{(n)} \mathbf{A}^{(n) T}+\mathbf{E}_{(n)} \mathbf{E}_{(n)}^{T}=\mathbf{U}^{(n)} \boldsymbol{\Sigma} \mathbf{U}^{(n) T}+\sigma^{2} I^{N-1} \mathbf{I}_{I_{n}} .
$$

where $\boldsymbol{\Sigma}=\mathbf{Q}\left(\mathbf{Q}^{T} \mathbf{Q}\right)^{\bullet[N-1]} \mathbf{Q}^{T},[\mathbf{A}]^{\bullet[p]}$ denotes element-wise power, and

$$
\sigma^{2}=\frac{\|y\|_{F}^{2}}{10^{\mathrm{SNR} / 10} I^{N}}=\frac{R^{2}+(R-1) x y-1}{10^{\mathrm{SNR} / 10} I^{N}}, \quad x=1+v^{2}, y=x^{N-1} .
$$

It is straightforward to prove that $\boldsymbol{\Sigma}=\left[\begin{array}{cc}R^{2}+(R-1)(y-1) & v(R+y-1) \mathbf{1}_{R-1}^{T} \\ v(R+y-1) \mathbf{1}_{R-1} & (x-1)\left(\mathbf{1}_{R-1} \mathbf{1}_{R-1}^{T}+(y-1) \mathbf{I}_{R-1}\right)\end{array}\right]$ has $(R-2)$ identical eigenvalues $\lambda_{r}=(x-1)(y-1), r=2, \ldots, R-1$, and its largest and smallest eigenvalues $\lambda_{1}>\lambda_{r}>\lambda_{R}$ are solutions of a quadratic equation

$$
\begin{aligned}
\lambda_{1}+\lambda_{R} & =x y+(R-2)(R+x+y)+3, \\
\lambda_{1} \lambda_{R} & =(x-1)(y-1)=\lambda_{r}, \quad 2 \leq r \leq R-1 .
\end{aligned}
$$

Fig.F.1(a) illustrates $\lambda_{r}(r=1, \ldots, R)$ for 3-D noiseless tensors with $I=100$ and $R=15$ compared with the noise levels $\sigma^{2} I^{N-1}$ at SNR $=20 \mathrm{~dB}$ and $30 \mathrm{~dB}$. The higher the collinearity degree of factor, the smaller the eigenvalues $\lambda_{r}$. If eigenvalues $\lambda_{r}$ are considerably lower than the noise level $\sigma^{2} I^{N-1}$, the factorization becomes infeasible, e.g., as $v \leq 0.1$.

Because $\mathbf{U}^{(n)}$ are orthonormal, $\mathbf{Y}_{(n)} \mathbf{Y}_{(n)}^{T}$ has $R$ leading eigenvalues $\tilde{\lambda}_{r}=\lambda_{r}+\sigma^{2} I^{(N-1)}, r=$ $1, \ldots, R$, and $(I-R)$ eigenvalues $\tilde{\lambda}_{i}=\sigma^{2} I^{(N-1)}, i=R+1, \ldots, I$. In Fig. F.1(b), we plot eigenvalues $\tilde{\lambda}_{i}$ for noisy tensors having the same dimension as that of tensors illustrated in Fig. F.1(a), The largest eigenvalue $\tilde{\lambda}_{1}$ significantly exceeds the noise levels, whereas $\tilde{\lambda}_{R}$ is quite close to the noise level at $\mathrm{SNR}=20 \mathrm{~dB}$ for $v \leq 0.3$, or at $\mathrm{SNR}=30 \mathrm{~dB}$ for $v \leq 0.1$. 


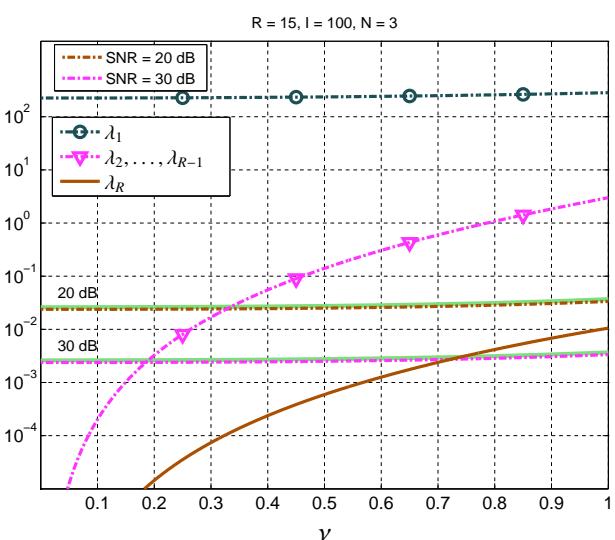

(a) Eigenvalues $\lambda_{r}, r=1, \ldots, R(=15), I_{n}=100, N=3$.

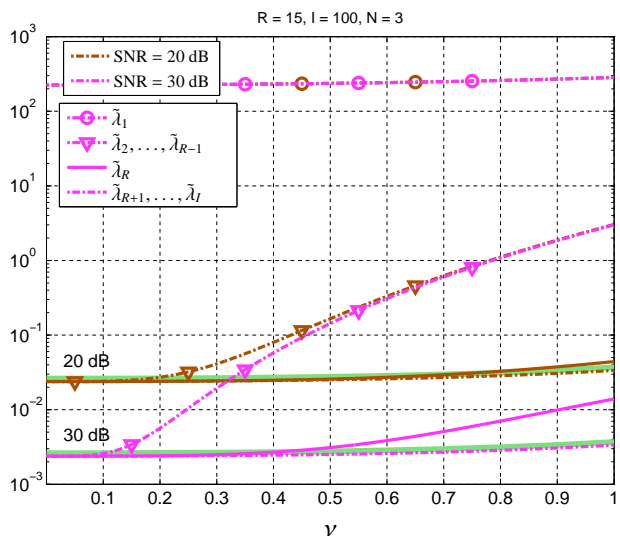

(b) Eigenvalues $\tilde{\lambda}_{i}, i=1, \ldots, I_{n}(=100), R=15, N=3$

FIG. F.1. Analysis of eigenvalues of $\mathbf{Y}_{(n)} \mathbf{Y}_{(n)}^{T}$ for 3-D tensors of size $I_{n}=100$ and rank $R=15$. $R$ leading eigenvalues $\lambda_{r}$ for noiseless tensors and $\tilde{\lambda}_{r}(r=1, \ldots, R)$ for noisy tensors are compared with noise levels (green shading) at $S N R=20 \mathrm{~dB}$ and $30 \mathrm{~dB}$. The more the eigenvalues are in the noise zone, the more difficult the factorization of noisy tensors to retrieve collinear factors become.

\section{REFERENCES}

[1] E. Acar, D. M. Dunlavy, and T. G. Kolda, A scalable optimization approach for fitting canonical tensor decompositions, Journal of Chemometrics, 25 (2011), pp. 67-86.

[2] C.A. Andersson and R. Bro, The N-way toolbox for MATLAB, Chemometrics Intell. Lab. Systems, 52 (2000), pp. 1-4.

[3] R. Bro, PARAFAC. Tutorial and applications, in Special Issue 2nd Internet Conf. in Chemometrics (INCINC'96), vol. 38, Chemom. Intell. Lab. Syst, 1997, pp. 149-171.

[4] J.D. Carroll and J.J. Chang, Analysis of individual differences in multidimensional scaling via an n-way generalization of Eckart-Young decomposition, Psychometrika, 35 (1970), pp. 283-319.

[5] A. Cichocki, R. Zdunek, A.-H. Phan, and S. Amari, Nonnegative Matrix and Tensor Factorizations: Applications to Exploratory Multi-way Data Analysis and Blind Source Separation, Wiley, Chichester, 2009.

[6] P. Comon, X. Luciani, AND A. L. F. DE Almeida, Tensor decompositions, alternating least squares and other tales, Jour. Chemometrics, 23 (2009), pp. 393-405.

[7] L. De Lathauwer, B. De Moor, and J. Vandewalle, On the best rank-1 and rank-(R1,R2,., RN) approximation of higher-order tensors, SIAM J. Matrix Anal. Appl., 21 (2000), pp. 1324-1342.

[8] P. Guillaume and R. Pintelon, A Gauss-Newton-like optimization algorithm for weighted nonlinear nonlinear least squares problems, IEEE Trans. Signal Processing, 44 (1996), pp. 2222-2228.

[9] X. Guo, S. Miron, D. Brie, and A. Stegeman, Uni-mode and partial uniqueness conditions for CANDECOMP/PARAFAC of three-way arrays with linearly dependent loadings, SIAM J. Matrix Anal. Appl., (2011).

[10] R.A. HaRShMan, Foundations of the PARAFAC procedure: Models and conditions for an explanatory multimodal factor analysis, UCLA Working Papers in Phonetics, 16 (1970), pp. 1-84.

[11] C. HaYashi and F. HaYashi, A new algorithm to solve PARAFAC-model, Behaviormetrika, 11 (1982), pp. 4960.

[12] F.L. Нгтснсоск, Multiple invariants and generalized rank of a p-way matrix or tensor, Journal of Mathematics and Physics, 7 (1927), pp. 39-79.

[13] R. A. Horn and C. R. Johnson, Matrix Analysis, Cambridge University Press, 1990.

[14] T.G. Kolda AND B.W. BADER, Tensor decompositions and applications, SIAM Review, 51 (2009), pp. 455-500.

[15] Z. KoldovskÝ, P. TichavskÝ, and A.-H. Phan, Stability analysis and fast damped Gauss-Newton algorithm for INDSCAL tensor decomposition, in Statistical Signal Processing Workshop (SSP), IEEE, 2011, pp. 581584.

[16] X.Q. Liu and N.D. Sidiropoulos, Cramer-Rao lower bounds for low-rank decomposition of multidimensional arrays, IEEE Transactions on Signal Processing, 49 (2001), pp. 2074-2086.

[17] B. C. Mitchell and D. S. Burdick, Slowly converging PARAFAC sequences: Swamps and two-factor degeneracies, Jour. Chemometrics, 8 (1994), pp. 155-168. 
[18] H. B. NIelsen, Damping parameter in Marquardt's method, tech. report, Department of Mathematical Modelling, DTU, 1999.

[19] P. PAATERo, Least-squares formulation of robust nonnegative factor analysis, Chemometrics and Intelligent Laboratory Systems, 37 (1997), pp. 23-35.

[20] — A weighted non-negative least squares algorithm for three-way PARAFAC factor analysis, Chemometrics Intelligent Laboratory Systems, 38 (1997), pp. 223-242.

[21] - The multilinear engine: A table-driven, least squares program for solving multilinear problems, including the n-way parallel factor analysis model, Journal of Computational and Graphical Statistics, 8 (1999), pp. 854-888.

[22] A.-H. Phan, P. TichavskÝ, and A. Cichocki, Damped Gauss-Newton algorithm for nonnegative Tucker decomposition, in Statistical Signal Processing Workshop (SSP), IEEE, 2011, pp. $665-668$.

[23] H. W. SoRenson, Parameter estimation: principles and problems, Marcel Dekker, NY, USA, 1980.

[24] P. Tichavský and Z. Koldovskŕ, Simultaneous search for all modes in multilinear models, in Proc. IEEE International Conference on Acoustics, Speech, and Signal Processing (ICASSP10), 2010, pp. 4114 4117.

[25] - Stability of CANDECOMP-PARAFAC tensor decomposition, in Proc. IEEE International Conference on Acoustics, Speech, and Signal Processing (ICASSP11), 2011, pp. 4164-4167.

[26] - Weight adjusted tensor method for blind separation of underdetermined mixtures of nonstationary sources, IEEE Transactions on Signal Processing, 59 (2011), pp. 1037-1047.

[27] P. TichavskÝ, A.-H. Phan, and Z. Koldovskŕ, Cramér-Rao induced bounds for CANDECOMP/PARAFAC tensor decomposition, (2012), p. submitted.

[28] G. Tomasi, INDAFAC and PARAFAC3W. http://www.models.kvl/dk/source/indafac/index.asp 2003.

[29] — Practical and Computational Aspects in Chemometric Data Analysis, PhD thesis, Frederiksberg, Denmark, 2006.

[30] - Recent developments in fast algorithms for fitting the PARAFAC model, in TRICAP 2006, Greece, 2006, TRICAP.

[31] G. Tomasi and R. Bro, PARAFAC and missing values, Chemometrics Intelligent Laboratory Systems, 75 (2005), pp. 163-180.

[32] - A comparison of algorithms for fitting the PARAFAC model, Computational Statistics and Data Analysis, 50 (2006), pp. 1700-1734.

[33] A. UschmaJEw, Local convergence of the alternating least squares algorithm for canonical tensor approximation, SIAM Journal on Matrix Analysis and Applications, 33 (2012), pp. 639-652. 